\title{
The Vertex-Edge Resolvability of Some Wheel-Related Graphs
}

\author{
Bao-Hua Xing, ${ }^{1}$ Sunny Kumar Sharma $\mathbb{D}^{2},{ }^{2}$ Vijay Kumar Bhat $\mathbb{D}^{2}{ }^{2}$ Hassan Raza, ${ }^{3}$ \\ and Jia-Bao Liu $\mathbb{D D}^{4}$ \\ ${ }^{1}$ School of Mathematics and Physics, Anqing Normal University, Anqing 246133, China \\ ${ }^{2}$ School of Mathematics, Faculty of Sciences, Shri Mata Vaishno Devi University, Katra 182320, Jammu and Kashmir, India \\ ${ }^{3}$ Business School, University of Shanghai for Science and Technology, Shanghai 200093, China \\ ${ }^{4}$ School of Mathematics and Physics, Anhui Jianzhu University, Hefei 230601, China
}

Correspondence should be addressed to Vijay Kumar Bhat; vijaykumarbhat2000@yahoo.com

Received 22 May 2021; Accepted 27 June 2021; Published 14 July 2021

Academic Editor: Ali Ahmad

Copyright ( 12021 Bao-Hua Xing et al. This is an open access article distributed under the Creative Commons Attribution License, which permits unrestricted use, distribution, and reproduction in any medium, provided the original work is properly cited.

A vertex $w \in V(H)$ distinguishes (or resolves) two elements (edges or vertices) $a, z \in V(H) \cup E(H)$ if $d(w, a) \neq d(w, z)$. A set $W_{m}$ of vertices in a nontrivial connected graph $H$ is said to be a mixed resolving set for $H$ if every two different elements (edges and vertices) of $H$ are distinguished by at least one vertex of $W_{m}$. The mixed resolving set with minimum cardinality in $H$ is called the mixed metric dimension (vertex-edge resolvability) of $H$ and denoted by $m \operatorname{dim}(H)$. The aim of this research is to determine the mixed metric dimension of some wheel graph subdivisions. We specifically analyze and compare the mixed metric, edge metric, and metric dimensions of the graphs obtained after the wheel graphs' spoke, cycle, and barycentric subdivisions. We also prove that the mixed resolving sets for some of these graphs are independent.

\section{Introduction}

Suppose $H=(V, E)$ is a nontrivial, simple, and connected graph, where $E$ represents a set of edges and $V$ represents a set of vertices. The distance between two vertices $a$ and $w$ in an undirected graph $H$, denoted by $d(a, w)$, is the length of a shortest $a-w$ path in $H$. In [1], Kelenc et al. introduced the concept of mixed metric dimension in graphs. This dimension of graph $H$ is the mixture of metric and edge metric dimensions.

A vertex $w \in V$ is said to resolve two vertices $v_{1}$ and $v_{2}$ in $H$ if $d\left(w, v_{1}\right) \neq d\left(w, v_{2}\right)$. Let $w$ be a vertex and $W=\left\{v_{1}, v_{2}, v_{3}, \ldots, v_{p}\right\}$ be an ordered subset of vertices in $H$. The metric coordinate (or metric representation) $r(w \mid W)$ of $w$ with respect to $W$ is the $p$-tuple $\left(d\left(w, v_{1}\right), d\left(w, v_{2}\right), d\left(w, v_{3}\right), \ldots, d\left(w, v_{p}\right)\right)$. Then, $W$ is said to be a resolving set (or metric generator) for $H$ if for every pair of vertices $v_{1}, v_{2} \in V$ with $v_{1} \neq v_{2}$, we have $r\left(v_{1} \mid W\right) \neq r\left(v_{2} \mid W\right)$. A resolving set with minimum cardinality is called the metric basis of $H$, and the cardinality of the metric basis set is the metric dimension $\operatorname{dim}(H)$ of $H$.
Slater introduced the idea of metric dimension in [2], where the metric generators were referred to as locating sets due to some relation with the problem of uniquely recognizing the location of intruders in networks. Harary and Melter, on the contrary, independently proposed the same concept of the metric dimension of a graph in [3], where metric generators were referred to as resolving sets. Several works on the applications and theoretical properties of this invariant have also been published. Metric dimension has various significant applications in computer science, mathematics, social sciences, chemical sciences, etc. [4-14]. There also exist some other variations of metric dimension in the literature: independent resolving sets [15], local metric dimension [16], solid metric dimension [11], fault-tolerant metric dimension [17], and so on.

The distance between an edge $e=a x$ and a vertex $w$ is defined as $d(e, w)=d(a x, w)=\min \{d(a, w), d(x, w)\}$. A vertex $w \in V$ is said to resolve two edges $e_{1}$ and $e_{2}$ in $H$ if $d\left(w, e_{1}\right) \neq d\left(w, e_{2}\right)$. Let $e$ be an edge and $W_{E}=$ $\left\{v_{1}, v_{2}, v_{3}, \ldots, v_{p}\right\}$ be an ordered subset of vertices in $H$. The edge metric codes $r_{E}\left(e \mid W_{E}\right)$ of $e$ with respect to $W_{E}$ are the 
p-tuple $\left(d\left(e, v_{1}\right), d\left(e, v_{2}\right), d\left(e, v_{3}\right), \ldots, d\left(e, v_{p}\right)\right)$. Then, $W_{E}$ is said to be an edge resolving set for $H$ if for every pair of edges $e_{1}, e_{2} \in E$ with $e_{1} \neq e_{2}$, we have $r_{E}\left(e_{1} \mid W_{E}\right)$ $\neq r_{E}\left(e_{2} \mid W_{E}\right)$. An edge resolving set with minimum cardinality is called an edge metric basis for $H$, and the cardinality of this edge metric basis set is the edge metric dimension $\operatorname{edim}(H)$ of $H$.

For a connected graph $H$, we see that every vertex of $H$ is uniquely recognized by a resolving set $W$ of $H$, and every edge of $H$ is uniquely recognized by an edge resolving set $W_{E}$ of $H$; the natural question is as follows: whether every resolving set $W$ is also an edge resolving set $W_{E}$ for $H$ and vice versa? Kelenc et al. in [18] proved that there exist some families of graphs for which the resolving set $W$ is also an edge resolving set $W_{E}$, but in general, this is not true for every graph $H$. Similarly, for every graph $H$, the edge resolving set is not necessarily a resolving set for $H$.

Let us define a set of elements as $V \cup E$, i.e., each element is an edge or a vertex. A vertex $w \in V$ is said to resolve two elements $a$ and $z$ from $V \cup E$ if $d(w, a) \neq d(w, z)$. Let $a$ be an element and $W_{m}=\left\{v_{1}, v_{2}, v_{3}, \ldots, v_{p}\right\}$ be an ordered subset of vertices in $H$. The mixed metric codes $r_{m}\left(a \mid W_{m}\right)$ of $a$ with respect to $W_{m}$ are the $p$-tuple $\left(d\left(a, v_{1}\right), d\left(a, v_{2}\right)\right.$, $\left.d\left(a, v_{3}\right), \ldots, d\left(a, v_{p}\right)\right)$. Then, $W_{m}$ is said to be a mixed resolving set for $H$ if for every pair of distinct elements $a_{1}, a_{2} \in V \cup E$, we have $r_{m}\left(a_{1} \mid W_{m}\right) \neq r_{m}\left(a_{2} \mid W_{m}\right)$. A mixed resolving set with minimum cardinality is called a mixed metric basis for $H$, and the cardinality of this mixed metric basis set is the mixed metric dimension $m \operatorname{dim}(H)$ of $H$. By the definition of the mixed metric dimension, it is clear that a mixed resolving set is both edge resolving set and a resolving set, so we have

$$
m \operatorname{dim}(H) \geq \max \{e \operatorname{dim}(H), \operatorname{dim}(H)\} .
$$

There are several studies $[1,19,20]$ related to the mixed metric dimension of various graphs, for instance, cycle graphs, antiprism graphs, prism graphs, and convex polytopes, but there are many graphs for which the mixed metric dimension has not been found yet, such as the graphs obtained by some subdivisions of the wheel graph $W_{n, 1}$. So, in this paper, we will compute the mixed metric dimension of the graphs obtained after the barycentric, spoke, and cycle subdivisions of the wheel graph $W_{n, 1}$.

\section{Preliminaries}

In this section, we give the definition of a wheel and its related graphs, as well as recall some existing results on the edge metric dimension, and the metric dimension of wheelrelated graphs.
2.1. Wheel Graph. A vertex $u$ in an undirected graph $G$ is said to be the universal vertex if it is adjacent to all other vertices of $G$. A wheel graph $W_{n, 1}(n \geq 3)$ is a graph with $n+1$ vertices obtained by joining a single universal vertex to all of the vertices of a cycle graph $C_{n}$. $W_{n, 1}$ has a vertex set $V=$ $\left\{v, k_{1}, k_{2}, k_{3}, \ldots, k_{n}\right\}$ and an edge set $E=\left\{v k_{j}, k_{j} k_{j+1}\right.$ $\mid 1 \leq j \leq n\}$, where all of the indices are taken to be modulo $n$. The edges $k_{j} k_{j+1}$ are called the cycle edges of $W_{n, 1}$, and the edges $v k_{j}$ are called as the spokes of the wheel graph.

We state that a family $F$ of nontrivial connected graphs has bounded mixed metric dimension if there exists a constant $L>0$ for every graph $H$ of $F$ such that $m \operatorname{dim}(H) \leq L$; otherwise, $\mathrm{F}$ has an unbounded mixed metric dimension. If all of the graphs in $F$ have the same mixed metric dimension, then $F$ is referred to as a family with a constant mixed metric dimension. Cycles $C_{n}$ and paths $P_{n}$ for $n \geq 3$ are the graph families with a constant mixed metric dimension.

2.2. Independent Mixed Resolving Set. A set $W_{m}$ of vertices from $H$ is said to be an independent mixed resolving set for $H$ if $W_{m}$ is an independent as well as mixed resolving set.

Let $\mathrm{WSS}_{n, 1}, \mathrm{WCS}_{n, 1}$, and $\mathrm{WBS}_{n, 1}$ be the graphs obtained from the wheel graph $W_{n, 1}$ after spoke, cycle, and barycentric subdivisions of $W_{n, 1}$, respectively. Recently, the metric and edge metric dimension for these three wheel-related graphs have been computed, and in [21], Raza and Bataineh made a comparison between the metric dimension and the edge metric dimension for these wheel-related graphs. The edge metric dimension and the metric dimension for these three graphs are as follows.

Proposition 1 (see [21]). edim $\left(W S S_{n, 1}\right)=n-1$, for $n \geq 6$.

Proposition 2 (see [21]). For $n \geq 6$, we have

$$
\begin{aligned}
e \operatorname{dim}\left(\mathrm{WCS}_{n, 1}\right)= & e \operatorname{dim}\left(\mathrm{WBS}_{n, 1}\right) \\
= & \begin{cases}4 h & \text { if } n=6 h \text { or } n=6 h+1 \\
4 h+1 & \text { if } n=6 h+2 \\
4 h+2 & \text { if } n=6 h+3 \text { or } n=6 h+4, \\
4 h+3 & \text { if } n=6 h+5\end{cases}
\end{aligned}
$$

Proposition 3 (see [22]). $\operatorname{dim}\left(W_{S S S_{n, 1}}\right)=\lfloor 2 n+2 / 5\rfloor$, for $n \geq 6$.

Proposition 4 (see $[23,22])$. For $n \geq 6$, we have

$$
\operatorname{dim}\left(\mathrm{WCS}_{n, 1}\right)=\operatorname{dim}\left(\mathrm{WBS}_{n, 1}\right)= \begin{cases}4 h & \text { if } n=6 h \text { or } n=6 h+1, \\ 4 h+1 & \text { if } n=6 h+2 \\ 4 h+2 & \text { if } n=6 h+3 \text { or } n=6 h+4 \\ 4 h+3 & \text { if } n=6 h+5\end{cases}
$$


This article is organized as follows: in Section 3, we will study the mixed metric dimension of the spoke subdivision of the wheel graph $\mathrm{WSS}_{n, 1}$. In Sections 4 and 5, we will study the mixed metric dimension of the cycle and barycentric subdivision of the wheel graph, i.e., $\mathrm{WCS}_{n, 1}$ and $\mathrm{WBS}_{n, 1}$, respectively. We also give the comparative analysis for the mixed metric, edge metric, and metric dimension of the graphs obtained after the spoke, cycle, and barycentric subdivisions of the wheel graph. In Section 6, we conclude the obtained results.

\section{Mixed Metric Dimension of the Spoke Subdivision of $W_{n, 1}$}

In this section, we determine the mixed metric dimension of the spoke subdivision of a wheel graph.

3.1. Spoke Subdivision of $W_{n, 1}$. Suppose $W_{n, 1}$ is a wheel graph with the vertex set $V\left(W_{n, 1}\right)=\left\{k_{1}, k_{2}, k_{3}, \ldots, k_{n}, v\right\}$ having a single universal vertex $v$. Now, each central spoke $v k_{j}$ of $W_{n, 1}$ is subdivided with a new vertex $l_{j}$. The resulting graph so obtained is known as the spoke subdivision wheel graph (SSWG) and is denoted by $\mathrm{WSS}_{n, 1}$. SSWG has $3 n$ edges, $E\left(W_{n, 1}\right)=\left\{v l_{j}, l_{j} k_{j}, k_{j} k_{j+1} \mid 1 \leq j \leq n\right\}$, and $2 n+1$ vertices, $V\left(W_{n, 1}\right)=\left\{v, l_{j}, k_{j} \mid 1 \leq j \leq n\right\}$, where all indices are taken to be modulo $n$ (see Figure 1). In this section, we obtain the mixed metric dimension of SSWG $\mathrm{WSS}_{n, 1}$.

Theorem 1. $m \operatorname{dim}\left(W S S_{n, 1}\right)=n$, for $n \geq 6$.

Proof. To prove that $m \operatorname{dim}\left(\mathrm{WSS}_{n, 1}\right) \leq n$, we construct a mixed resolving set for $\mathrm{WSS}_{n, 1}$. Suppose $W_{m}=\left\{k_{1}, k_{2}, k_{3}, \ldots, k_{n}\right\} \subseteq V\left(\mathrm{WSS}_{n, 1}\right)$ having $n$ cycle vertices from $\mathrm{WSS}_{n, 1}$. We claim that $W_{m}$ is a mixed resolving set for $\mathrm{WSS}_{n, 1}$. Now, we can give mixed codes to each of the vertex and edge of $\mathrm{WSS}_{n, 1}$ with respect to $W_{m}$.

The sets of mixed metric codes for the vertices $\left\{v, l_{j}, k_{j} \mid 1 \leq j \leq n\right\}$ of $\mathrm{WSS}_{n, 1}$ are as follows:

$$
\begin{aligned}
& A=\{r_{m}\left(v \mid W_{m}\right)=\underbrace{(2,2,2, \ldots, 2)}_{n \text {-times }}\}, \\
& B=\left\{r_{m}\left(l_{j} \mid W_{m}\right)=(3,3, \ldots, 3,2, \underbrace{1}_{j^{\text {th }}}, 2,3, \ldots, 3,3) \mid 1 \leq j \leq n\right\}, \\
& C=\left\{r_{m}\left(k_{j} \mid W_{m}\right)=(4,4, \ldots, 4,3,2,1, \underbrace{0}_{j^{\text {th }}}, 1,2,3,4, \ldots, 4,4) \mid 1 \leq j \leq n\right\} .
\end{aligned}
$$

Next, the sets of mixed metric codes for the edges $\left\{v l_{j}, l_{j} k_{j}, k_{j} k_{j+1} \mid 1 \leq j \leq n\right\}$ of $\mathrm{WSS}_{n, 1}$ are as follows:

$$
\begin{aligned}
& D=\left\{r_{m}\left(v l_{j} \mid W_{m}\right)=(2,2, \ldots, 2, \underbrace{1}_{j^{\mathrm{th}}}, 2, \ldots, 2,2) \mid 1 \leq j \leq n\right\}, \\
& E=\left\{r_{m}\left(l_{j} k_{j} \mid W_{m}\right)=(3,3, \ldots, 3,2,1, \underbrace{0}_{j^{\mathrm{th}}}, 1,2,3, \ldots, 3,3) \mid 1 \leq j \leq n\right\}, \\
& F=\left\{r_{m}\left(k_{j} k_{j+1} \mid W_{m}\right)=(4,4, \ldots, 4,3,2,1, \underbrace{0}_{j^{\mathrm{th}}}, 0,1,2,3,4, \ldots, 4,4) \mid 1 \leq j \leq n\right\} .
\end{aligned}
$$

From these sets of mixed codes for WSS $_{n, 1}$, we obtain that $|A|=1,|B|=|C|=|D|=|E|=|F|=n$, and $A \cap B \cap$ $C \cap D \cap E \cap F=\varnothing$, implying $W_{m}$ to be a mixed resolving set for $\mathrm{WSS}_{n, 1}$, i.e., $m \operatorname{dim}\left(\mathrm{WSS}_{n, 1}\right) \leq n$. Conversely, suppose, on the contrary, that there exists a mixed resolving set $W_{m} \subseteq W_{S S}$ such that $\left|W_{m}\right|<n$. Then, we have the following cases to be considered:

Case (i): $v \notin W_{m}$. In this case, we further have two subcases:
Subcase (i): if $W_{m} \subset\left\{k_{1}, k_{2}, k_{3}, \ldots, k_{n}\right\}$, then there exists at least one vertex $k_{j}$ such that $k_{j} \notin W_{m}$. Then, for an edge $v l_{j}$ and the vertex $v$, we have $r_{m}\left(v l_{j} \mid W_{m}\right)=r_{m}\left(v \mid W_{m}\right)=(2,2,2, \ldots, 2)$, a contradiction. Therefore, the set $W_{m}$ is not a mixed resolving set for $\mathrm{WSS}_{n, 1}$.

Subcase (ii): if $W_{m} \nsubseteq\left\{k_{1}, k_{2}, k_{3}, \ldots, k_{n}\right\}$, then at least one vertex $l_{i}$ belongs to the set $W_{m}$. Then, there exists one $k_{j} \notin W_{m}$, and the corresponding vertex $l_{j} \notin W_{m}$. Then, for an edge $v l_{j}$ and the vertex $v$, we have 


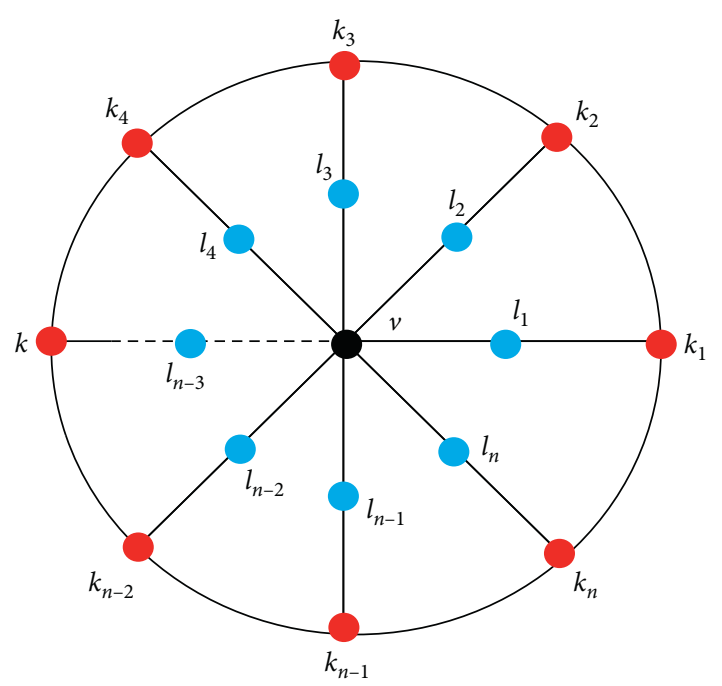

FIgURE 1: $\mathrm{WSS}_{n, 1}$.

$r_{m}\left(v l_{j} \mid W_{m}\right)=r_{m}\left(v \mid W_{m}\right)$, a contradiction. Therefore, again, in this case, the set $W_{m}$ is not a mixed resolving set for $\mathrm{WSS}_{n, 1}$.

Case (ii): $v \in W_{m}$. In this case, we have two subcases:

Subcase (i): if $W_{m} \subset\left\{k_{1}, k_{2}, k_{3}, \ldots, k_{n}\right\} \cup\{v\}$, then there exists at least one vertex $k_{j}$ such that $k_{j} \notin W_{m}$. Then, clearly, for an edge $v l_{j}$ and the vertex $v$, we have $r_{m}\left(v l_{j} \mid W_{m}\right)=r_{m}\left(v \mid W_{m}\right)$, a contradiction. Therefore, the set $W_{m}$ is not a mixed resolving set for $\mathrm{WSS}_{n, 1}$.

Subcase (ii): if at least one $l_{j}$ must belong to the set $W_{m}$, then there exists at least one vertex $k_{j} \notin W_{m}$, and the corresponding vertex $l_{j} \notin W_{m}$. Then, for an edge $v l_{j}$ and a vertex $v$, we have $r_{m}\left(v l_{j} \mid W_{m}\right)=r_{m}\left(v \mid W_{m}\right)$, a contradiction. Therefore, again, in this case, the set $W_{m}$ is not a mixed resolving set for $\mathrm{WSS}_{n, 1}$. Thus, in all the cases, we have $\left|W_{m}\right| \geq n$, implying $m \operatorname{dim}\left(\mathrm{WSS}_{n, 1}\right)$ $=n$, which completes the proof of the theorem.

Remark 1. For the spoke subdivision wheel graph $H=\mathrm{WSS}_{n, 1}$, we find that $\operatorname{dim}\left(\mathrm{WSS}_{n, 1}\right)<e \operatorname{dim}\left(\mathrm{WSS}_{n, 1}\right)<$ $m \operatorname{dim}\left(\mathrm{WSS}_{n, 1}\right)$ (using Propositions 1 and 3 and Theorem 1). The comparison between these three dimensions of WSS $_{n, 1}$ is clearly shown in Figure 2, and the value of each dimension depends on the number of vertices $n$ in WSS $_{n, 1}$.

\section{Mixed Metric Dimension of the Cycle Subdivision of $W_{n, 1}$}

In this section, we determine the mixed metric dimension of the cycle subdivision of a wheel graph.

4.1. Cycle Subdivision of $W_{n, 1}$. Suppose $W_{n, 1}$ is a wheel graph with the vertex set $V\left(W_{n, 1}\right)=\left\{k_{1}, k_{2}, k_{3}, \ldots, k_{n}, v\right\}$ having a single universal vertex $v$. Now, each cycle edge $k_{j} k_{j+1}$ of $W_{n, 1}$ is subdivided with a new vertex $l_{j}$. The resulting graph so obtained is known as the cycle subdivision wheel graph (CSWG) and is denoted by $\mathrm{WCS}_{n, 1}$. CSWG has $3 n$ edges, $E\left(\mathrm{WCS}_{n, 1}\right)=\left\{v k_{j}, k_{j} l_{j}, l_{j} k_{j+1} \mid 1 \leq j \leq n\right\}$, and $2 n+1$ vertices, $V\left(\mathrm{WCS}_{n, 1}\right)=\left\{v, l_{j}, k_{j} \mid 1 \leq j \leq n\right\}$, where all indices are taken to be modulo $n$ (see Figure 3 ). In this section, we obtain the mixed metric dimension of CSWG $\mathrm{WCS}_{n, 1}$.

Theorem 2. For $n \geq 6$, we have

$$
m \operatorname{dim}\left(\mathrm{WCS}_{n, 1}\right)= \begin{cases}4 h & \text { if } n=6 h, \\ 4 h+1 & \text { if } n=6 h+1, \\ 4 h+2 & \text { if } n=6 h+2, \\ 4 h+2 & \text { if } n=6 h+3, \\ 4 h+3 & \text { if } n=6 h+4 \\ 4 h+4 & \text { if } n=6 h+5\end{cases}
$$

Proof. To prove this, we first generate the mixed resolving sets for all the cases, obtaining the upper bounds depending on the positive integer $n$. Then, in the end, we show that the lower bound (or reverse inequality) is the same as the upper bound to conclude the theorem.

Case $(\mathrm{I}): n \equiv 0(\bmod 6)$. In this case, we have $n=6 h$, where $h \geq 2$ and $h \in \mathbb{N}$. Suppose an ordered subset $W_{m}=\left\{l_{1}, l_{2}, l_{4}, l_{5}, \ldots, l_{n-2}, l_{n-1}\right\}=\left\{l_{3 i+1}, l_{3 i+2} \mid 0 \leq i \leq 2 h\right.$ $-1\}$ of vertices in $\mathrm{WCS}_{n, 1}$ with $\left|W_{m}\right|=4 h$. Next, we claim that $W_{m}$ is the mixed resolving set for $\mathrm{WCS}_{n, 1}$. Now, we can give mixed codes to every vertex and edge of $\mathrm{WCS}_{n, 1}$ with respect to $W_{m}$. The sets of mixed metric codes for the vertices $\left\{u=v, l_{j}, k_{j} \mid 1 \leq j \leq n\right\}$ of $\operatorname{WCS}_{n, 1}$ are as follows:

$$
\begin{aligned}
A= & \{r_{m}\left(v \mid W_{m}\right)=\underbrace{(2,2,2, \ldots, 2)}_{4 h \text {-times }}\}, \\
B= & \left\{\begin{array}{c}
r_{m}\left(k_{j} \mid W_{m}\right)=\left(3,3,3, \ldots, 3, d\left(l_{3 i+2}, k_{3 i+3}\right)=1,3, \ldots, 3\right) \mid \\
j \equiv 0(\bmod 3) 0 \leq i \leq 2 h-1
\end{array}\right\} \cup \\
& \left\{\begin{array}{c}
r_{m}\left(k_{j} \mid W_{m}\right)=\left(3,3,3, \ldots, 3, d\left(l_{3 i+1}, k_{3 i+1}\right)=1,3, \ldots, 3\right) \mid \\
j \equiv 1(\bmod 3) 0 \leq i \leq 2 h-1
\end{array}\right\} \cup
\end{aligned}
$$




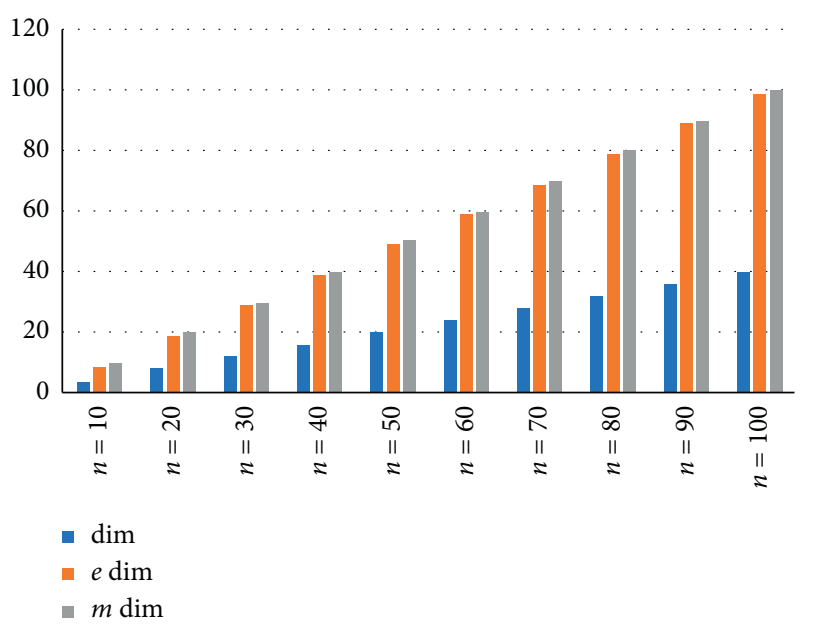

Figure 2: Comparison between $\operatorname{dim}(H), e \operatorname{dim}(H)$, and $m \operatorname{dim}(H)$.

$$
\begin{gathered}
\left\{\begin{array}{c}
r_{m}\left(k_{j} \mid W_{m}\right)=\left(3,3,3, \ldots, 3, d\left(l_{3 i+1}, k_{3 i+2}\right)=1, d\left(l_{3 i+2}, k_{3 i+2}\right)=1,3, \ldots, 3\right) \mid \\
j \equiv 2(\bmod 3) 0 \leq i \leq 2 h-1
\end{array}\right\}, \\
C=\left\{\begin{array}{c}
r_{m}\left(l_{j} \mid W_{m}\right)=\left(4,, 4, \ldots, 4, d\left(l_{3 i+2}, l_{3 i+3}\right)=2, d\left(l_{3 i+4}, l_{3 i+3}\right)=2,4, \ldots, 4\right) \mid \\
j \equiv 0(\bmod 3) 0 \leq i \leq 2 h-1
\end{array}\right\} \cup \\
\\
\left\{\begin{array}{c}
r_{m}\left(l_{j} \mid W_{m}\right)=\left(4,4,4, \ldots, 4, d\left(l_{3 i+1}, l_{3 i+1}\right)=0, d\left(l_{3 i+1}, l_{3 i+2}\right)=2,4, \ldots, 4\right) \mid \\
j \equiv 1(\bmod 3) 0 \leq i \leq 2 h-1
\end{array}\right\} \cup \\
\left\{\begin{array}{c}
r_{m}\left(l_{j} \mid W_{m}\right)=\left(4,4,4, \ldots, 4, d\left(l_{3 i+1}, l_{3 i+1}\right)=2, d\left(l_{3 i+1}, l_{3 i+2}\right)=0,4, \ldots, 4\right) \mid \\
j \equiv 2(\bmod 3) 0 \leq i \leq 2 h-1
\end{array}\right\} .
\end{gathered}
$$

Next, the sets of mixed metric codes for the edges $\left\{v k_{j}, k_{j} l_{j}, l_{j} k_{j+1} \mid 1 \leq j \leq n\right\}$ of $\mathrm{WCS}_{n, 1}$ are as follows:

$$
\begin{aligned}
D= & \left\{r_{m}\left(v k_{j} \mid W_{m}\right)=\left(2,2,2, \ldots, 2, d\left(l_{3 i+2}, v k_{3 i+3}\right)=1,2, \ldots, 2\right) \mid j \equiv 0(\bmod 3) \& 0 \leq i \leq 2 h-1\right\} \\
& \cup\left\{r_{m}\left(v k_{j} \mid W_{m}\right)=\left(2,2,2, \ldots, 2, d\left(l_{3 i+1}, v k_{3 i+2}\right)=1,2, \ldots, 2\right) \mid j \equiv 1(\bmod 3) \& 0 \leq i \leq 2 h-1\right\} \\
& \cup\left\{r_{m}\left(v k_{j} \mid W_{m}\right)=\left(2,2,2, \ldots, 2, d\left(l_{3 i+1}, v k_{3 i+2}\right)=1, d\left(l_{3 i+2}, v k_{3 i+2}\right)=1,2, \ldots, 2\right) \mid j \equiv 2(\bmod 3) \& 0 \leq i \leq 2 h-1\right\} ; \\
E=\{ & \left\{r_{m}\left(k_{j} l_{j} \mid W_{m}\right)=\left(3,3,3, \ldots, 3, d\left(l_{3 i+2}, k_{3 i+3} l_{3 i+3}\right)=1, d\left(l_{3 i+4}, k_{3 i+3} l_{3 i+3}\right)=2,3, \ldots, 3\right) \mid j \equiv 0(\bmod 3) \& 0 \leq i \leq 2 h-1\right\} \\
& \cup\left\{r_{m}\left(k_{j} l_{j} \mid W_{m}\right)=\left(3,3,3, \ldots, 3, d\left(l_{3 i+1}, k_{3 i+1} l_{3 i+1}\right)=0, d\left(l_{3 i+2}, k_{3 i+1} l_{3 i+1}\right)=2,3, \ldots, 3\right) \mid j \equiv 1(\bmod 3) \& 0 \leq i \leq 2 h-1\right\} \\
& \cup\left\{r_{m}\left(k_{j} l_{j} \mid W_{m}\right)=\left(3,3,3, \ldots, 3, d\left(l_{3 i+1}, k_{3 i+2} l_{3 i+2}\right)=1, d\left(l_{3 i+2}, k_{3 i+2} l_{3 i+2}\right)=0,3, \ldots, 3\right) \mid j \equiv 2(\bmod 3) \& 0 \leq i \leq 2 h-1\right\} ; \\
F=\{ & \left\{r_{m}\left(l_{j} k_{j+1} \mid W_{m}\right)=\left(3,3,3, \ldots, 3, d\left(l_{3 i+2}, l_{3 i+3} k_{3 i+4}\right)=2, d\left(l_{3 i+4}, l_{3 i+3} k_{3 i+4}\right)=1,3, \ldots, 3\right) \mid j \equiv 0(\bmod 3) \& 0 \leq i \leq 2 h-1\right\} \\
& \cup\left\{r_{m}\left(l_{j} k_{j+1} \mid W_{m}\right)=\left(3,3,3, \ldots, 3, d\left(l_{3 i+1}, l_{3 i+1} k_{3 i+2}\right)=0, d\left(l_{3 i+2}, l_{3 i+1} k_{3 i+2}\right)=1,3, \ldots, 3\right) \mid j \equiv 1(\bmod 3) \& 0 \leq i \leq 2 h-1\right\} \\
& \cup\left\{r_{m}\left(l_{j} k_{j+1} \mid W_{m}\right)=\left(3,3,3, \ldots, 3, d\left(l_{3 i+1}, l_{3 i+2} k_{3 i+3}\right)=2, d\left(l_{3 i+2}, l_{3 i+2} k_{3 i+3}\right)=0,3, \ldots, 3\right) \mid j \equiv 2(\bmod 3) \& 0 \leq i \leq 2 h-1\right\} .
\end{aligned}
$$




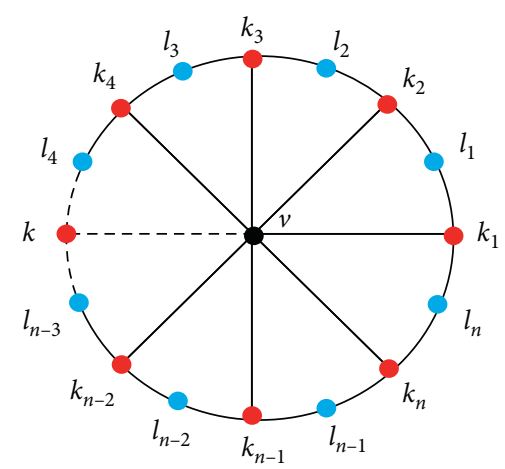

FIGURE 3: $\mathrm{WCS}_{n, 1}$.

From these sets of mixed codes for $\mathrm{WCS}_{n, 1}$, we obtain that $|A|=1, \quad|B|=|C|=|D|=|E|=|F|=n$, and $A \cap B \cap C \cap D \cap E \cap F=\varnothing$, implying $W_{m}$ to be a mixed resolving set for $\mathrm{WCS}_{n, 1}$, i.e., $m \operatorname{dim}\left(\mathrm{WCS}_{n, 1}\right) \leq 4 h$. Next, using equation (1) and Proposition 2, we find that $m \operatorname{dim}\left(\mathrm{WCS}_{n, 1}\right)=4 h$, in this case.

Case (II): $n \equiv 1(\bmod 6)$. In this case, we have $n=6 h+1$, where $h \geq 2$ and $h \in \mathbb{N}$. Suppose an ordered subset $W_{m}=\left\{l_{1}, l_{2}, l_{4}, l_{5}, \ldots, l_{n-3}, l_{n-2}, l_{n}\right\}=\left\{l_{3 i+1}, l_{3 i+2}\right.$ $\mid 0 \leq i \leq 2 h-1\} \cup\left\{l_{n}\right\}$ of vertices in $\mathrm{WCS}_{n, 1}$ with $\left|W_{m}\right|=4 h+1$. Next, we claim that $W_{m}$ is the mixed resolving set for $\mathrm{WCS}_{n, 1}$. Now, we can give mixed codes to every vertex and edge of $\mathrm{WCS}_{n, 1}$ with respect to $W_{m}$. The sets of mixed metric codes for the vertices $\left\{u=v, l_{j}, k_{j} \mid 1 \leq j \leq n\right\}$ of $\mathrm{WCS}_{n, 1}$ are as follows:

$$
\begin{aligned}
& A=\{r_{m}\left(v \mid W_{m}\right)=\underbrace{(2,2,2, \ldots, 2)}_{(4 h+1)-\text { times }}\} \\
& B=\left\{\begin{array}{c}
r_{m}\left(k_{j} \mid W_{m}\right)=\left(3,3,3, \ldots, 3, d\left(l_{3 i+2}, k_{3 i+3}\right)=1,3, \ldots, 3\right) \mid \\
j \equiv 0(\bmod 3) 0 \leq i \leq 2 h-1
\end{array}\right\} \cup \\
& \left\{r_{m}\left(k_{1} \mid W_{m}\right)=(1, \underbrace{3,3, \ldots, 3}_{(4 h-1)-\text { times }}, 1)\right\} U \\
& \left\{\begin{array}{c}
r_{m}\left(k_{j} \mid W_{m}\right)=\left(3,3,3, \ldots, 3, d\left(l_{3 i+1}, k_{3 i+1}\right)=1,3, \ldots, 3\right) \mid \\
j \equiv 1(\bmod 3) 1 \leq i \leq 2 h
\end{array}\right\} \cup \\
& \left\{\begin{array}{c}
r_{m}\left(k_{j} \mid W_{m}\right)=\left(3,3,3, \ldots, 3, d\left(l_{3 i+1}, k_{3 i+2}\right)=1, d\left(l_{3 i+2}, k_{3 i+2}\right)=1,3, \ldots, 3\right) \mid \\
j \equiv 2(\bmod 3) 0 \leq i \leq 2 h-1
\end{array}\right\}, \\
& C=\left\{\begin{array}{c}
r_{m}\left(l_{j} \mid W_{m}\right)=\left(4,, 4, \ldots, 4, d\left(l_{3 i+2}, l_{3 i+3}\right)=2, d\left(l_{3 i+4}, l_{3 i+3}\right)=2,4, \ldots, 4\right) \mid \\
j \equiv 0(\bmod 3) 0 \leq i \leq 2 h-1
\end{array}\right\} \cup
\end{aligned}
$$




$$
\begin{aligned}
& \left\{r_{m}\left(l_{1} \mid W_{m}\right)=(0,2, \underbrace{4,4,4, \ldots, 4}_{(4 h-2) \text {-times }}, 2)\right\} \cup \\
& \left\{\begin{array}{c}
r_{m}\left(l_{j} \mid W_{m}\right)=\left(4,4,4, \ldots, 4, d\left(l_{3 i+1}, l_{3 i+1}\right)=0, d\left(l_{3 i+1}, l_{3 i+2}\right)=2,4, \ldots, 4\right) \mid \\
j \equiv 1(\bmod 3) 1 \leq i \leq 2 h
\end{array}\right\} \cup \\
& \left\{\begin{array}{c}
r_{m}\left(l_{j} \mid W_{m}\right)=\left(4,4,4, \ldots, 4, d\left(l_{3 i+1}, l_{3 i+1}\right)=2, d\left(l_{3 i+1}, l_{3 i+2}\right)=0,4, \ldots, 4\right) \mid \\
j \equiv 2(\bmod 3) 0 \leq i \leq 2 h-1
\end{array}\right\} .
\end{aligned}
$$

Next, the sets of mixed metric codes for the edges $\left\{v k_{j}, k_{j} l_{j}, l_{j} k_{j+1} \mid 1 \leq j \leq n\right\}$ of $\mathrm{WCS}_{n, 1}$ are as follows:

$$
\begin{aligned}
& D=\left\{\begin{array}{c}
r_{m}\left(v k_{j} \mid W_{m}\right)=\left(2,2,2, \ldots, 2, d\left(l_{3 i+2}, v k_{3 i+3}\right)=1,2, \ldots, 2\right) \mid \\
j \equiv 0(\bmod 3) 0 \leq i \leq 2 h-1
\end{array}\right\} \cup \\
& \left\{r_{m}\left(v k_{1} \mid W_{m}\right)=(1, \underbrace{2,2,2, \ldots, 2}_{(4 h-1) \text {-times }}, 1)\right\} U \\
& \left\{\begin{array}{c}
r_{m}\left(v k_{j} \mid W_{m}\right)=\left(2,2,2, \ldots, 2, d\left(l_{3 i+1}, v k_{3 i+1}\right)=1,2, \ldots, 2\right) \mid \\
j \equiv 1(\bmod 3) 1 \leq i \leq 2 h
\end{array}\right\} \cup \\
& \left\{\begin{array}{c}
r_{m}\left(v k_{j} \mid W_{m}\right)=\left(2,2,2, \ldots, 2, d\left(l_{3 i+1}, v k_{3 i+2}\right)=1, d\left(l_{3 i+2}, v k_{3 i+2}\right)=1,2, \ldots, 2\right) \mid \\
j \equiv 2(\bmod 3) 0 \leq i \leq 2 h-1
\end{array}\right\}, \\
& E=\left\{\begin{array}{c}
r_{m}\left(k_{j} l_{j} \mid W_{m}\right)=\left(3,3,3, \ldots, 3, d\left(l_{3 i+2}, k_{3 i+3} l_{3 i+3}\right)=1, d\left(l_{3 i+4}, k_{3 i+3} l_{3 i+3}\right)=2,3, \ldots, 3\right) \mid \\
j \equiv 0(\bmod 3) 0 \leq i \leq 2 h-1
\end{array}\right\} \cup \\
& \left\{r_{m}\left(k_{1} l_{1} \mid W_{m}\right)=(0,2, \underbrace{3,3,3, \ldots, 3}_{(4 h-2) \text {-times }}, 1)\right\} U \\
& \left\{\begin{array}{c}
r_{m}\left(k_{j} l_{j} \mid W_{m}\right)=\left(3,3,3, \ldots, 3, d\left(l_{3 i+1}, k_{3 i+1} l_{3 i+1}\right)=0, d\left(l_{3 i+2}, k_{3 i+1} l_{3 i+1}\right)=2,3, \ldots, 3\right) \mid \\
j \equiv 1(\bmod 3) 1 \leq i \leq 2 h
\end{array}\right\} \cup \\
& \left\{\begin{array}{c}
r_{m}\left(k_{j} l_{j} \mid W_{m}\right)=\left(3,3,3, \ldots, 3, d\left(l_{3 i+1}, k_{3 i+2} l_{3 i+2}\right)=1, d\left(l_{3 i+2}, k_{3 i+2} l_{3 i+2}\right)=0,3, \ldots, 3\right) \mid \\
j \equiv 2(\bmod 3) 0 \leq i \leq 2 h-1
\end{array}\right\}, \\
& F=\left\{\begin{array}{c}
r_{m}\left(l_{j} k_{j+1} \mid W_{m}\right)=\left(3,3,3, \ldots, 3, d\left(l_{3 i+2}, l_{3 i+3} k_{3 i+4}\right)=2, d\left(l_{3 i+4}, l_{3 i+3} k_{3 i+4}\right)=1,3, \ldots, 3\right) \mid \\
j \equiv 0(\bmod 3) 0 \leq i \leq 2 h-1
\end{array}\right\} \cup \\
& \left\{r_{m}\left(l_{1} k_{2} \mid W_{m}\right)=(0,1, \underbrace{3,3,3, \ldots, 3}_{(4 h-2) \text {-times }}, 1)\right\} \cup \\
& \left\{\begin{array}{c}
r_{m}\left(l_{j} k_{j+1} \mid W_{m}\right)=\left(3,3,3, \ldots, 3, d\left(l_{3 i+1}, l_{3 i+1} k_{3 i+2}\right)=0, d\left(l_{3 i+2}, l_{3 i+1} k_{3 i+2}\right)=1,3, \ldots, 3\right) \mid \\
j \equiv 1(\bmod 3) 1 \leq i \leq 2 h
\end{array}\right\} \cup \\
& \left\{\begin{array}{c}
r_{m}\left(l_{j} k_{j+1} \mid W_{m}\right)=\left(3,3,3, \ldots, 3, d\left(l_{3 i+1}, l_{3 i+2} k_{3 i+3}\right)=2, d\left(l_{3 i+2}, l_{3 i+2} k_{3 i+3}\right)=0,3, \ldots, 3\right) \mid \\
j \equiv 2(\bmod 3) 0 \leq i \leq 2 h-1
\end{array}\right\} .
\end{aligned}
$$


From these sets of mixed codes for $\mathrm{WCS}_{n, 1}$, we obtain that $|A|=1, \quad|B|=|C|=|D|=|E|=|F|=n$, and $A \cap B \cap C \cap D \cap E \cap F=\varnothing$, implying $W_{m}$ to be a mixed resolving set for $\mathrm{WCS}_{n, 1}$, i.e., $m \operatorname{dim}\left(\mathrm{WCS}_{n, 1}\right) \leq 4 h+1$.

Case (III): $n \equiv 2(\bmod 6)$. In this case, we have $n=6 h+2$, where $h \geq 2$ and $h \in \mathbb{N}$. Suppose an ordered subset $W_{m}=\left\{l_{1}, l_{2}, l_{4}, l_{5}, l_{7} \ldots, l_{n-1}, l_{n}\right\}=\left\{l_{3 i+1}, l_{3 i+2} \mid 0\right.$ $\leq i \leq 2 h\}$ of vertices in $\mathrm{WCS}_{n, 1}$ with $\left|W_{m}\right|=4 h+2$. Next, we claim that $W_{m}$ is the mixed resolving set for $\mathrm{WCS}_{n, 1}$. Now, we can give mixed codes to every vertex and edge of $\mathrm{WCS}_{n, 1}$ with respect to $W_{m}$. The sets of mixed metric codes for the vertices $\left\{u=v, l_{j}, k_{j} \mid 1 \leq j \leq n\right\}$ of $\mathrm{WCS}_{n, 1}$ are as follows:

$$
\begin{aligned}
& A=\{r_{m}\left(v \mid W_{m}\right)=\underbrace{(2,2,2, \ldots, 2)}_{(4 h+2) \text {-times }}\}, \\
& B=\left\{\begin{array}{c}
r_{m}\left(k_{j} \mid W_{m}\right)=\left(3,3,3, \ldots, 3, d\left(l_{3 i+2}, k_{3 i+3}\right)=1,3, \ldots, 3\right) \mid \\
j \equiv 0(\bmod 3) 0 \leq i \leq 2 h-1
\end{array}\right\} \cup \\
& \left\{r_{m}\left(k_{1} \mid W_{m}\right)=(1, \underbrace{3,3, \ldots, 3}_{(4 h) \text {-times }}, 1)\right\} U \\
& \left\{\begin{array}{c}
r_{m}\left(k_{j} \mid W_{m}\right)=\left(3,3,3, \ldots, 3, d\left(l_{3 i+1}, k_{3 i+1}\right)=1,3, \ldots, 3\right) \mid \\
j \equiv 1(\bmod 3) 1 \leq i \leq 2 h
\end{array}\right\} \cup \\
& \left\{\begin{array}{c}
r_{m}\left(k_{j} \mid W_{m}\right)=\left(3,3,3, \ldots, 3, d\left(l_{3 i+1}, k_{3 i+2}\right)=1, d\left(l_{3 i+2}, k_{3 i+2}\right)=1,3, \ldots, 3\right) \mid \\
j \equiv 2(\bmod 3) 0 \leq i \leq 2 h
\end{array}\right\}, \\
& C=\left\{\begin{array}{c}
r_{m}\left(l_{j} \mid W_{m}\right)=\left(4,, 4, \ldots, 4, d\left(l_{3 i+2}, l_{3 i+3}\right)=2, d\left(l_{3 i+4}, l_{3 i+3}\right)=2,4, \ldots, 4\right) \mid \\
j \equiv 0(\bmod 3) 0 \leq i \leq 2 h-1
\end{array}\right\} \cup \\
& \left\{r_{m}\left(l_{1} \mid W_{m}\right)=(0,2, \underbrace{4,4,4, \ldots, 4}_{(4 h-1) \text {-times }}, 2)\right\} \cup \\
& \left\{\begin{array}{c}
r_{m}\left(l_{j} \mid W_{m}\right)=\left(4,4,4, \ldots, 4, d\left(l_{3 i+1}, l_{3 i+1}\right)=0, d\left(l_{3 i+1}, l_{3 i+2}\right)=2,4, \ldots, 4\right) \mid \\
j \equiv 1(\bmod 3) 1 \leq i \leq 2 h
\end{array}\right\} \cup \\
& \left\{\begin{array}{c}
r_{m}\left(l_{j} \mid W_{m}\right)=\left(4,4,4, \ldots, 4, d\left(l_{3 i+1}, l_{3 i+2}\right)=2, d\left(l_{3 i+2}, l_{3 i+2}\right)=0,4, \ldots, 4\right) \mid \\
j \equiv 2(\bmod 3) 0 \leq i \leq 2 h
\end{array}\right\} .
\end{aligned}
$$

Next, the sets of mixed metric codes for the edges

$\left\{v k_{j}, k_{j} l_{j}, l_{j} k_{j+1} \mid 1 \leq j \leq n\right\}$ of $\mathrm{WCS}_{n, 1}$ are as follows:

$$
\begin{aligned}
D= & \left\{\begin{array}{c}
r_{m}\left(v k_{j} \mid W_{m}\right)=\left(2,2,2, \ldots, 2, d\left(l_{3 i+2}, v k_{3 i+3}\right)=1,2, \ldots, 2\right) \mid \\
j \equiv 0(\bmod 3) 0 \leq i \leq 2 h-1
\end{array}\right\} U \\
& \left\{r_{m}\left(v k_{1} \mid W_{m}\right)=(1, \underbrace{2,2,2, \ldots, 2}_{(4 h)-\text { times }}, 1)\right\} \cup \\
& \left\{\begin{array}{c}
r_{m}\left(v k_{j} \mid W_{m}\right)=\left(2,2,2, \ldots, 2, d\left(l_{3 i+1}, v k_{3 i+1}\right)=1,2, \ldots, 2\right) \mid \\
j \equiv 1(\bmod 3) 1 \leq i \leq 2 h
\end{array}\right\} U
\end{aligned}
$$




$$
\begin{aligned}
& \left\{\begin{array}{c}
r_{m}\left(v k_{j} \mid W_{m}\right)=\left(2,2,2, \ldots, 2, d\left(l_{3 i+1}, v k_{3 i+2}\right)=1, d\left(l_{3 i+2}, v k_{3 i+2}\right)=1,2, \ldots, 2\right) \mid \\
j \equiv 2(\bmod 3) 0 \leq i \leq 2 h
\end{array}\right\}, \\
& E=\left\{\begin{array}{c}
r_{m}\left(k_{j} l_{j} \mid W_{m}\right)=\left(3,3,3, \ldots, 3, d\left(l_{3 i+2}, k_{3 i+3} l_{3 i+3}\right)=1, d\left(l_{3 i+4}, k_{3 i+3} l_{3 i+3}\right)=2,3, \ldots, 3\right) \mid \\
j \equiv 0(\bmod 3) 0 \leq i \leq 2 h-1
\end{array}\right\} \cup \\
& \left\{r_{m}\left(k_{1} l_{1} \mid W_{m}\right)=(0,2, \underbrace{3,3,3, \ldots, 3}_{(4 h-1) \text {-times }}, 1)\right\} U \\
& \left\{\begin{array}{c}
r_{m}\left(k_{j} l_{j} \mid W_{m}\right)=\left(3,3,3, \ldots, 3, d\left(l_{3 i+1}, k_{3 i+1} l_{3 i+1}\right)=0, d\left(l_{3 i+2}, k_{3 i+1} l_{3 i+1}\right)=2,3, \ldots, 3\right) \mid \\
j \equiv 1(\bmod 3) 1 \leq i \leq 2 h
\end{array}\right\} \cup \\
& \left\{\begin{array}{c}
r_{m}\left(k_{j} l_{j} \mid W_{m}\right)=\left(3,3,3, \ldots, 3, d\left(l_{3 i+1}, k_{3 i+2} l_{3 i+2}\right)=1, d\left(l_{3 i+2}, k_{3 i+2} l_{3 i+2}\right)=0,3, \ldots, 3\right) \mid \\
j \equiv 2(\bmod 3) 0 \leq i \leq 2 h
\end{array}\right\}, \\
& F=\left\{\begin{array}{c}
r_{m}\left(l_{j} k_{j+1} \mid W_{m}\right)=\left(3,3,3, \ldots, 3, d\left(l_{3 i+2}, l_{3 i+3} k_{3 i+4}\right)=2, d\left(l_{3 i+4}, l_{3 i+3} k_{3 i+4}\right)=1,3, \ldots, 3\right) \mid \\
j \equiv 0(\bmod 3) 0 \leq i \leq 2 h-1
\end{array}\right\} \cup \\
& \left\{r_{m}\left(l_{1} k_{2} \mid W_{m}\right)=(0,1, \underbrace{3,3,3, \ldots, 3}_{(4 h-1) \text {-times }}, 1)\right\} \cup \\
& \left\{\begin{array}{c}
r_{m}\left(l_{j} k_{j+1} \mid W_{m}\right)=\left(3,3,3, \ldots, 3, d\left(l_{3 i+1}, l_{3 i+1} k_{3 i+2}\right)=0, d\left(l_{3 i+2}, l_{3 i+1} k_{3 i+2}\right)=1,3, \ldots, 3\right) \mid \\
j \equiv 1(\bmod 3) 1 \leq i \leq 2 h
\end{array}\right\} \cup \\
& \left\{\begin{array}{c}
r_{m}\left(l_{j} k_{j+1} \mid W_{m}\right)=\left(3,3,3, \ldots, 3, d\left(l_{3 i+1}, l_{3 i+2} k_{3 i+3}\right)=2, d\left(l_{3 i+2}, l_{3 i+2} k_{3 i+3}\right)=0,3, \ldots, 3\right) \mid \\
j \equiv 2(\bmod 3) 0 \leq i \leq 2 h
\end{array}\right\} .
\end{aligned}
$$

From these sets of mixed codes for $\mathrm{WCS}_{n, 1}$, we obtain that $|A|=1, \quad|B|=|C|=|D|=|E|=|F|=n$, and $A \cap B \cap C \cap D \cap E \cap F=\varnothing$, implying $W_{m}$ to be a mixed resolving set for $\mathrm{WCS}_{n, 1}$, i.e., $m \operatorname{dim}\left(\mathrm{WCS}_{n, 1}\right) \leq 4 h+2$.

Case (IV): $n \equiv 3(\bmod 6)$. In this case, we have $n=6 h+3$, where $h \geq 2$ and $h \in \mathbb{N}$. Suppose an ordered subset $W_{m}=\left\{l_{1}, l_{2}, l_{4}, l_{5}, l_{7} \ldots, l_{n-2}, l_{n-1}\right\}=\left\{l_{3 i+1}, l_{3 i+2} \mid\right.$
$0 \leq i \leq 2 h\}$ of vertices in $\mathrm{WCS}_{n, 1}$ with $\left|W_{m}\right|=4 h+2$. Next, we claim that $W_{m}$ is the mixed resolving set for $\mathrm{WCS}_{n, 1}$. Now, we can give mixed codes to every vertex and edge of $\mathrm{WCS}_{n, 1}$ with respect to $W_{m}$. The sets of mixed metric codes for the vertices $\left\{u=v, l_{j}, k_{j} \mid 1 \leq j \leq n\right\}$ of $\mathrm{WCS}_{n, 1}$ are as follows:

$$
\begin{aligned}
A= & \{r_{m}\left(v \mid W_{m}\right)=\underbrace{(2,2,2, \ldots, 2)}_{(4 h+2) \text {-times }}\}, \\
B= & \left\{\begin{array}{c}
r_{m}\left(k_{j} \mid W_{m}\right)=\left(3,3,3, \ldots, 3, d\left(l_{3 i+2}, k_{3 i+3}\right)=1,3, \ldots, 3\right) \mid \\
j \equiv 0(\bmod 3) 0 \leq i \leq 2 h
\end{array}\right\} \cup \\
& \left\{\begin{array}{c}
r_{m}\left(k_{j} \mid W_{m}\right)=\left(3,3,3, \ldots, 3, d\left(l_{3 i+1}, k_{3 i+1}\right)=1,3, \ldots, 3\right) \mid \\
j \equiv 1(\bmod 3) 0 \leq i \leq 2 h
\end{array}\right\} \cup \\
& \left\{\begin{array}{c}
r_{m}\left(k_{j} \mid W_{m}\right)=\left(3,3,3, \ldots, 3, d\left(l_{3 i+1}, k_{3 i+2}\right)=1, d\left(l_{3 i+2}, k_{3 i+2}\right)=1,3, \ldots, 3\right) \mid \\
j \equiv 2(\bmod 3) 0 \leq i \leq 2 h
\end{array}\right\}, \\
C= & \left\{\begin{array}{c}
r_{m}\left(l_{j} \mid W_{m}\right)=\left(4,, 4, \ldots, 4, d\left(l_{3 i+2}, l_{3 i+3}\right)=2, d\left(l_{3 i+4}, l_{3 i+3}\right)=2,4, \ldots, 4\right) \mid \\
j \equiv 0(\bmod 3) 0 \leq i \leq 2 h
\end{array}\right\} \cup \\
& \left\{\begin{array}{c}
r_{m}\left(l_{j} \mid W_{m}\right)=\left(4,4,4, \ldots, 4, d\left(l_{3 i+1}, l_{3 i+1}\right)=0, d\left(l_{3 i+1}, l_{3 i+2}\right)=2,4, \ldots, 4\right) \mid \\
j \equiv 1(\bmod 3) 0 \leq i \leq 2 h
\end{array}\right\} \cup \\
& \left\{\begin{array}{c}
r_{m}\left(l_{j} \mid W_{m}\right)=\left(4,4,4, \ldots, 4, d\left(l_{3 i+1}, l_{3 i+2}\right)=2, d\left(l_{3 i+2}, l_{3 i+2}\right)=0,4, \ldots, 4\right) \mid \\
j \equiv 2(\bmod 3) 0 \leq i \leq 2 h
\end{array}\right\} .
\end{aligned}
$$


Next, the sets of mixed metric codes for the edges

$\left\{v k_{j}, k_{j} l_{j}, l_{j} k_{j+1} \mid 1 \leq j \leq n\right\}$ of $\mathrm{WCS}_{n, 1}$ are as follows:

$$
\begin{aligned}
& D=\left\{r_{m}\left(v k_{j} \mid W_{m}\right)=\left(2,2,2, \ldots, 2, d\left(l_{3 i+2}, v k_{3 i+3}\right)=1,2, \ldots, 2\right) \mid j \equiv 0(\bmod 3) \& 0 \leq i \leq 2 h\right\} \\
& \cup\left\{r_{m}\left(v k_{j} \mid W_{m}\right)=\left(2,2,2, \ldots, 2, d\left(l_{3 i+1}, v k_{3 i+2}\right)=1,2, \ldots, 2\right) \mid j \equiv 1(\bmod 3) \& 0 \leq i \leq 2 h\right\} \\
& \cup\left\{r_{m}\left(v k_{j} \mid W_{m}\right)=\left(2,2,2, \ldots, 2, d\left(l_{3 i+1}, v k_{3 i+2}\right)=1, d\left(l_{3 i+2}, v k_{3 i+2}\right)=1,2, \ldots, 2\right) \mid j \equiv 2(\bmod 3) \& 0 \leq i \leq 2 h\right\} ; \\
& E=\left\{r_{m}\left(k_{j} l_{j} \mid W_{m}\right)=\left(3,3,3, \ldots, 3, d\left(l_{3 i+2}, k_{3 i+3} l_{3 i+3}\right)=1, d\left(l_{3 i+4}, k_{3 i+3} l_{3 i+3}\right)=2,3, \ldots, 3\right) \mid j \equiv 0(\bmod 3) \& 0 \leq i \leq 2 h\right\} \\
& \cup\left\{r_{m}\left(k_{j} l_{j} \mid W_{m}\right)=\left(3,3,3, \ldots, 3, d\left(l_{3 i+1}, k_{3 i+1} l_{3 i+1}\right)=0, d\left(l_{3 i+2}, k_{3 i+1} l_{3 i+1}\right)=2,3, \ldots, 3\right) \mid j \equiv 1(\bmod 3) \& 0 \leq i \leq 2 h\right\} \\
& \cup\left\{r_{m}\left(k_{j} l_{j} \mid W_{m}\right)=\left(3,3,3, \ldots, 3, d\left(l_{3 i+1}, k_{3 i+2} l_{3 i+2}\right)=1, d\left(l_{3 i+2}, k_{3 i+2} l_{3 i+2}\right)=0,3, \ldots, 3\right) \mid j \equiv 2(\bmod 3) \& 0 \leq i \leq 2 h\right\} ; \\
& F=\left\{r_{m}\left(l_{j} k_{j+1} \mid W_{m}\right)=\left(3,3,3, \ldots, 3, d\left(l_{3 i+2}, l_{3 i+3} k_{3 i+4}\right)=2, d\left(l_{3 i+4}, l_{3 i+3} k_{3 i+4}\right)=1,3, \ldots, 3\right) \mid j \equiv 0(\bmod 3) \& 0 \leq i \leq 2 h\right\} \\
& \cup\left\{r_{m}\left(l_{j} k_{j+1} \mid W_{m}\right)=\left(3,3,3, \ldots, 3, d\left(l_{3 i+1}, l_{3 i+1} k_{3 i+2}\right)=0, d\left(l_{3 i+2}, l_{3 i+1} k_{3 i+2}\right)=1,3, \ldots, 3\right) \mid j \equiv 1(\bmod 3) \& 0 \leq i \leq 2 h\right\} . \\
& \cup\left\{r_{m}\left(l_{j} k_{j+1} \mid W_{m}\right)=\left(3,3,3, \ldots, 3, d\left(l_{3 i+1}, l_{3 i+2} k_{3 i+3}\right)=2, d\left(l_{3 i+2}, l_{3 i+2} k_{3 i+3}\right)=0,3, \ldots, 3\right) \mid j \equiv 2(\bmod 3) \& 0 \leq i \leq 2 h\right\} ;
\end{aligned}
$$

From these sets of mixed codes for $\mathrm{WCS}_{n, 1}$, we obtain that $|A|=1, \quad|B|=|C|=|D|=|E|=|F|=n$, and $A \cap B \cap C \cap D \cap E \cap F=\varnothing$, implying $W_{m}$ to be a mixed resolving set for $\mathrm{WCS}_{n, 1}$, i.e., $m \operatorname{dim}\left(\mathrm{WCS}_{n, 1}\right) \leq 4 h+2$. Next, using equation (1) and Proposition 2, we find that $m \operatorname{dim}\left(\mathrm{WCS}_{n, 1}\right)=4 h+2$, in this case.

Case $(\mathrm{V}): n \equiv 4(\bmod 6)$. In this case, we have $n=6 h+4$, where $h \geq 2$ and $h \in \mathbb{N}$. Suppose an ordered subset $W_{m}=\left\{l_{1}, l_{2}, l_{4}, l_{5}, \ldots, l_{n-3}, l_{n-2}, l_{n}\right\}=\left\{l_{3 i+1}, l_{3 i+2} \mid\right.$ $0 \leq i \leq 2 h\} \cup\left\{l_{n}\right\} \quad$ of vertices in WCS $_{n, 1}$ with $\left|W_{m}\right|=4 h+3$. Next, we claim that $W_{m}$ is the mixed resolving set for $\mathrm{WCS}_{n, 1}$. Now, we can give mixed codes to every vertex and edge of $\mathrm{WCS}_{n, 1}$ with respect to $W_{m}$. The sets of mixed metric codes for the vertices $\left\{u=v, l_{j}, k_{j} \mid 1 \leq j \leq n\right\}$ of $\mathrm{WCS}_{n, 1}$ are as follows:

$$
\begin{aligned}
A= & \{r_{m}\left(v \mid W_{m}\right)=\underbrace{(2,2,2, \ldots, 2)}_{(4 h+3) \text {-times }}\}, \\
B= & \left\{\begin{array}{c}
r_{m}\left(k_{j} \mid W_{m}\right)=\left(3,3,3, \ldots, 3, d\left(l_{3 i+2}, k_{3 i+3}\right)=1,3, \ldots, 3\right) \mid \\
j \equiv 0(\bmod 3) 0 \leq i \leq 2 h
\end{array}\right\} \cup \\
& \left\{r_{m}\left(k_{1} \mid W_{m}\right)=(1, \underbrace{3,3, \ldots, 3}_{(4 h+1) \text {-times }}, 1)\right\} \cup \\
& \left\{r_{m}\left(k_{j} \mid W_{m}\right)=\left(3,3,3, \ldots, 3, d\left(l_{3 i+1}, k_{3 i+1}\right)=1,3, \ldots, 3\right) \mid j \equiv 1(\bmod 3) 1 \leq i \leq 2 h+1\right\} \cup \\
& \left\{\begin{array}{c}
r_{m}\left(k_{j} \mid W_{m}\right)=\left(3,3,3, \ldots, 3, d\left(l_{3 i+1}, k_{3 i+2}\right)=1, d\left(l_{3 i+2}, k_{3 i+2}\right)=1,3, \ldots, 3\right) \mid \\
j \equiv 2(\bmod 3) 0 \leq i \leq 2 h+1
\end{array},\right.
\end{aligned}
$$




$$
\begin{aligned}
C= & \left\{\begin{array}{c}
r_{m}\left(l_{j} \mid W_{m}\right)=\left(4,, 4, \ldots, 4, d\left(l_{3 i+2}, l_{3 i+3}\right)=2, d\left(l_{3 i+4}, l_{3 i+3}\right)=2,4, \ldots, 4\right) \mid \\
j \equiv 0(\bmod 3) 0 \leq i \leq 2 h
\end{array}\right\} \\
& \cup\left\{r_{m}\left(l_{1} \mid W_{m}\right)=(0,2, \underbrace{4,4, \ldots, 4}_{(4 h)-\operatorname{4imes}}, 2)\right\} \cup \\
& \left\{\begin{array}{c}
r_{m}\left(l_{j} \mid W_{m}\right)=\left(4,4,4, \ldots, 4, d\left(l_{3 i+1}, l_{3 i+1}\right)=0, d\left(l_{3 i+1}, l_{3 i+2}\right)=2,4, \ldots, 4\right) \mid \\
j \equiv 1(\bmod 3) 1 \leq i \leq 2 h+1
\end{array}\right\} \cup \\
& \left\{\begin{array}{c}
r_{m}\left(l_{j} \mid W_{m}\right)=\left(4,4,4, \ldots, 4, d\left(l_{3 i+1}, l_{3 i+1}\right)=2, d\left(l_{3 i+1}, l_{3 i+2}\right)=0,4, \ldots, 4\right) \mid \\
j \equiv 2(\bmod 3) 0 \leq i \leq 2 h+1
\end{array}\right\} .
\end{aligned}
$$

Next, the sets of mixed metric codes for the edges

$\left\{v k_{j}, k_{j} l_{j}, l_{j} k_{j+1} \mid 1 \leq j \leq n\right\}$ of $\mathrm{WCS}_{n, 1}$ are as follows:

$$
\begin{aligned}
& D=\left\{\begin{array}{c}
r_{m}\left(v k_{j} \mid W_{m}\right)=\left(2,2,2, \ldots, 2, d\left(l_{3 i+2}, v k_{3 i+3}\right)=1,2, \ldots, 2\right) \mid \\
j \equiv 0(\bmod 3) 0 \leq i \leq 2 h
\end{array}\right\} U \\
& \left\{r_{m}\left(v k_{1} \mid W_{m}\right)=(1, \underbrace{2,2,2, \ldots, 2}_{(4 h+1)-\text { times }}, 1)\right\} \cup \\
& \left\{\begin{array}{c}
r_{m}\left(v k_{j} \mid W_{m}\right)=\left(2,2,2, \ldots, 2, d\left(l_{3 i+1}, v k_{3 i+1}\right)=1,2, \ldots, 2\right) \mid \\
j \equiv 1(\bmod 3) 1 \leq i \leq 2 h+1
\end{array}\right\} \cup \\
& \left\{\begin{array}{c}
r_{m}\left(v k_{j} \mid W_{m}\right)=\left(2,2,2, \ldots, 2, d\left(l_{3 i+1}, v k_{3 i+2}\right)=1, d\left(l_{3 i+2}, v k_{3 i+2}\right)=1,2, \ldots, 2\right) \mid \\
j \equiv 2(\bmod 3) 0 \leq i \leq 2 h
\end{array}\right\}, \\
& E=\left\{\begin{array}{c}
r_{m}\left(k_{j} l_{j} \mid W_{m}\right)=\left(3,3,3, \ldots, 3, d\left(l_{3 i+2}, k_{3 i+3} l_{3 i+3}\right)=1, d\left(l_{3 i+4}, k_{3 i+3} l_{3 i+3}\right)=2,3, \ldots, 3\right) \mid \\
j \equiv 0(\bmod 3) 0 \leq i \leq 2 h
\end{array}\right\} \cup \\
& \left\{r_{m}\left(k_{1} l_{1} \mid W_{m}\right)=(0,2, \underbrace{3,3,3, \ldots, 3}_{(4 h) \text {-times }}, 1)\right\} U \\
& \left\{\begin{array}{c}
r_{m}\left(k_{j} l_{j} \mid W_{m}\right)=\left(3,3,3, \ldots, 3, d\left(l_{3 i+1}, k_{3 i+1} l_{3 i+1}\right)=0, d\left(l_{3 i+2}, k_{3 i+1} l_{3 i+1}\right)=2,3, \ldots, 3\right) \mid \\
j \equiv 1(\bmod 3) 1 \leq i \leq 2 h+1
\end{array}\right\} \cup \\
& \left\{\begin{array}{c}
r_{m}\left(k_{j} l_{j} \mid W_{m}\right)=\left(3,3,3, \ldots, 3, d\left(l_{3 i+1}, k_{3 i+2} l_{3 i+2}\right)=1, d\left(l_{3 i+2}, k_{3 i+2} l_{3 i+2}\right)=0,3, \ldots, 3\right) \mid \\
j \equiv 2(\bmod 3) 0 \leq i \leq 2 h+1
\end{array}\right\}, \\
& F=\left\{\begin{array}{c}
r_{m}\left(l_{j} k_{j+1} \mid W_{m}\right)=\left(3,3,3, \ldots, 3, d\left(l_{3 i+2}, l_{3 i+3} k_{3 i+4}\right)=2, d\left(l_{3 i+4}, l_{3 i+3} k_{3 i+4}\right)=1,3, \ldots, 3\right) \mid \\
j \equiv 0(\bmod 3) 0 \leq i \leq 2 h
\end{array}\right\} \cup \\
& \left\{r_{m}\left(l_{1} k_{2} \mid W_{m}\right)=(0,1, \underbrace{3,3,3, \ldots, 3}_{(4 h)-\text { times }}, 1)\right\} U \\
& \left\{\begin{array}{c}
r_{m}\left(l_{j} k_{j+1} \mid W_{m}\right)=\left(3,3,3, \ldots, 3, d\left(l_{3 i+1}, l_{3 i+1} k_{3 i+2}\right)=0, d\left(l_{3 i+2}, l_{3 i+1} k_{3 i+2}\right)=1,3, \ldots, 3\right) \mid \\
j \equiv 1(\bmod 3) 1 \leq i \leq 2 h+1
\end{array}\right\} \cup \\
& \left\{\begin{array}{c}
r_{m}\left(l_{j} k_{j+1} \mid W_{m}\right)=\left(3,3,3, \ldots, 3, d\left(l_{3 i+1}, l_{3 i+2} k_{3 i+3}\right)=2, d\left(l_{3 i+2}, l_{3 i+2} k_{3 i+3}\right)=0,3, \ldots, 3\right) \mid \\
j \equiv 2(\bmod 3) 0 \leq i \leq 2 h
\end{array}\right\} .
\end{aligned}
$$


From these sets of mixed codes for $\mathrm{WCS}_{n, 1}$, we obtain that $|A|=1,|B|=|C|=|D|=|E|=|F|=n$, and $A \cap B \cap C \cap D \cap E \cap F=\varnothing$, implying $W_{m}$ to be a mixed resolving set for $\mathrm{WCS}_{n, 1}$, i.e., $m \operatorname{dim}\left(\mathrm{WCS}_{n, 1}\right) \leq 4 h+3$.

Case (VI): $n \equiv 5(\bmod 6)$. In this case, we have $n=6 h+5$, where $h \geq 1$ and $h \in \mathbb{N}$. Suppose an ordered subset $\quad W_{m}=\left\{l_{1}, l_{2}, l_{4}, l_{5}, \ldots, l_{n-1}, l_{n}\right\}=\left\{l_{3 i+1}, l_{3 i+2} \mid\right.$ $0 \leq i \leq 2 h+1\}$ of vertices in $\mathrm{WCS}_{n, 1}$ with $\left|W_{m}\right|=4 h+4$. Next, we claim that $W_{m}$ is the mixed resolving set for $\mathrm{WCS}_{n, 1}$. Now, we can give mixed codes to every vertex and edge of $\mathrm{WCS}_{n, 1}$ with respect to $W_{m}$. The sets of mixed metric codes for the vertices $\left\{u=v, l_{j}, k_{j} \mid 1 \leq j \leq n\right\}$ of $\operatorname{WCS}_{n, 1}$ are as follows:

$$
\begin{aligned}
A= & \{r_{m}\left(v \mid W_{m}\right)=\underbrace{(2,2,2, \ldots, 2)}_{(4 h+4) \text {-times }}\}, \\
B= & \left\{\begin{array}{c}
r_{m}\left(k_{j} \mid W_{m}\right)=\left(3,3,3, \ldots, 3, d\left(l_{3 i+2}, k_{3 i+3}\right)=1,3, \ldots, 3\right) \mid \\
j \equiv 0(\bmod 3) 0 \leq i \leq 2 h
\end{array}\right\} \cup \\
& \left\{r_{m}\left(k_{1} \mid W_{m}\right)=(1, \underbrace{3,3, \ldots, 3}_{(4 h+2)-\operatorname{times}}, 1)\right\} \\
& \left\{\begin{array}{c}
r_{m}\left(k_{j} \mid W_{m}\right)=\left(3,3,3, \ldots, 3, d\left(l_{3 i+1}, k_{3 i+1}\right)=1,3, \ldots, 3\right) \mid \\
j \equiv 1(\bmod 3) 1 \leq i \leq 2 h+1
\end{array}\right\} \cup \\
& \left\{\begin{array}{c}
r_{m}\left(k_{j} \mid W_{m}\right)=\left(3,3,3, \ldots, 3, d\left(l_{3 i+1}, k_{3 i+2}\right)=1, d\left(l_{3 i+2}, k_{3 i+2}\right)=1,3, \ldots, 3\right) \mid \\
j \equiv 2(\bmod 3) 0 \leq i \leq 2 h+1
\end{array}\right\}, \\
C= & \left\{\begin{array}{c}
r_{m}\left(l_{j} \mid W_{m}\right)=\left(4,, 4, \ldots, 4, d\left(l_{3 i+2}, l_{3 i+3}\right)=2, d\left(l_{3 i+4}, l_{3 i+3}\right)=2,4, \ldots, 4\right) \mid \\
j \equiv 0(\bmod 3) 0 \leq i \leq 2 h
\end{array}\right\} \cup \\
& \left\{\begin{array}{c}
\left.r_{m}\left(l_{1} \mid W_{m}\right)=(0,2,4,4,4, \ldots, 4,2)\right\} \cup \\
(4 h+1) \text {-times }
\end{array}\right\} \\
& \left\{\begin{array}{c}
r_{m}\left(l_{j} \mid W_{m}\right)=\left(4,4,4, \ldots, 4, d\left(l_{3 i+1}, l_{3 i+1}\right)=0, d\left(l_{3 i+1}, l_{3 i+2}\right)=2,4, \ldots, 4\right) \mid \\
j \equiv 1(\bmod 3) 1 \leq i \leq 2 h+1
\end{array}\right\} \cup \\
& \left\{\begin{array}{c}
r_{m}\left(l_{j} \mid W_{m}\right)=\left(4,4,4, \ldots, 4, d\left(l_{3 i+1}, l_{3 i+2}\right)=2, d\left(l_{3 i+2}, l_{3 i+2}\right)=0,4, \ldots, 4\right) \mid \\
j \equiv 2(\bmod 3) 0 \leq i \leq 2 h+1
\end{array}\right\}
\end{aligned}
$$

Next, the sets of mixed metric codes for the edges

$\left\{v k_{j}, k_{j} l_{j}, l_{j} k_{j+1} \mid 1 \leq j \leq n\right\}$ of $\mathrm{WCS}_{n, 1}$ are as follows:

$$
\begin{aligned}
D= & \left\{\begin{array}{c}
r_{m}\left(v k_{j} \mid W_{m}\right)=\left(2,2,2, \ldots, 2, d\left(l_{3 i+2}, v k_{3 i+3}\right)=1,2, \ldots, 2\right) \mid \\
j \equiv 0(\bmod 3) 0 \leq i \leq 2 h
\end{array}\right\} \cup \\
& \left\{r_{m}\left(v k_{1} \mid W_{m}\right)=\left(\begin{array}{c}
1,2,2,2, \ldots, 2 \\
(4 h+2)-\operatorname{times}
\end{array}\right)\right\} \cup \\
& \left\{\begin{array}{c}
r_{m}\left(v k_{j} \mid W_{m}\right)=\left(2,2,2, \ldots, 2, d\left(l_{3 i+1}, v k_{3 i+1}\right)=1,2, \ldots, 2\right) \mid \\
j \equiv 1(\bmod 3) 1 \leq i \leq 2 h+1
\end{array}\right\} \cup \\
& \left\{\begin{array}{c}
r_{m}\left(v k_{j} \mid W_{m}\right)=\left(2,2,2, \ldots, 2, d\left(l_{3 i+1}, v k_{3 i+2}\right)=1, d\left(l_{3 i+2}, v k_{3 i+2}\right)=1,2, \ldots, 2\right) \mid \\
j \equiv 2(\bmod 3) 0 \leq i \leq 2 h+1
\end{array}\right\}, \\
E= & \left\{\begin{array}{c}
r_{m}\left(k_{j} l_{j} \mid W_{m}\right)=\left(3,3,3, \ldots, 3, d\left(l_{3 i+2}, k_{3 i+3} l_{3 i+3}\right)=1, d\left(l_{3 i+4}, k_{3 i+3} l_{3 i+3}\right)=2,3, \ldots, 3\right) \mid \\
j \equiv 0(\bmod 3) 0 \leq i \leq 2 h
\end{array}\right\} U
\end{aligned}
$$




$$
\begin{aligned}
& \left\{r_{m}\left(k_{1} l_{1} \mid W_{m}\right)=(0,2, \underbrace{3,3,3, \ldots, 3}_{(4 h+1) \text {-times }}, 1)\right\} U \\
& \left\{\begin{array}{c}
r_{m}\left(k_{j} l_{j} \mid W_{m}\right)=\left(3,3,3, \ldots, 3, d\left(l_{3 i+1}, k_{3 i+1} l_{3 i+1}\right)=0, d\left(l_{3 i+2}, k_{3 i+1} l_{3 i+1}\right)=2,3, \ldots, 3\right) \mid \\
j \equiv 1(\bmod 3) 1 \leq i \leq 2 h+1
\end{array}\right\} \cup \\
& \left\{\begin{array}{c}
r_{m}\left(k_{j} l_{j} \mid W_{m}\right)=\left(3,3,3, \ldots, 3, d\left(l_{3 i+1}, k_{3 i+2} l_{3 i+2}\right)=1, d\left(l_{3 i+2}, k_{3 i+2} l_{3 i+2}\right)=0,3, \ldots, 3\right) \mid \\
j \equiv 2(\bmod 3) 0 \leq i \leq 2 h+1
\end{array}\right\}, \\
& F=\left\{\begin{array}{c}
r_{m}\left(l_{j} k_{j+1} \mid W_{m}\right)=\left(3,3,3, \ldots, 3, d\left(l_{3 i+2}, l_{3 i+3} k_{3 i+4}\right)=2, d\left(l_{3 i+4}, l_{3 i+3} k_{3 i+4}\right)=1,3, \ldots, 3\right) \mid \\
j \equiv 0(\bmod 3) 0 \leq i \leq 2 h
\end{array}\right\} \cup \\
& \left\{r_{m}\left(l_{1} k_{2} \mid W_{m}\right)=(0,1, \underbrace{3,3,3, \ldots, 3}_{(4 h+1) \text {-times }}, 2)\right\} \cup \\
& \left\{\begin{array}{c}
r_{m}\left(l_{j} k_{j+1} \mid W_{m}\right)=\left(3,3,3, \ldots, 3, d\left(l_{3 i+1}, l_{3 i+1} k_{3 i+2}\right)=0, d\left(l_{3 i+2}, l_{3 i+1} k_{3 i+2}\right)=1,3, \ldots, 3\right) \mid \\
j \equiv 1(\bmod 3) 1 \leq i \leq 2 h+1
\end{array}\right\} \cup \\
& \left\{\begin{array}{c}
r_{m}\left(l_{j} k_{j+1} \mid W_{m}\right)=\left(3,3,3, \ldots, 3, d\left(l_{3 i+1}, l_{3 i+2} k_{3 i+3}\right)=2, d\left(l_{3 i+2}, l_{3 i+2} k_{3 i+3}\right)=0,3, \ldots, 3\right) \mid \\
j \equiv 2(\bmod 3) 0 \leq i \leq 2 h+1
\end{array}\right\} .
\end{aligned}
$$

From these sets of mixed codes for $\mathrm{WCS}_{n, 1}$, we obtain that $|A|=1, \quad|B|=|C|=|D|=|E|=|F|=n$, and $A \cap B \cap C \cap D \cap E \cap F=\varnothing$, implying $W_{m}$ to be a mixed resolving set for $\mathrm{WCS}_{n, 1}$, i.e., $m \operatorname{dim}\left(\mathrm{WCS}_{n, 1}\right) \leq 4 h+4$. Now, for the second, third, fifth, and sixth case, we obtain their lower bounds as follows.

For the second case, suppose that $W_{m} \subset V\left(\mathrm{WCS}_{n, 1}\right)$ with $\left|W_{m}\right|<4 h+1$ is a mixed resolving set for $\mathrm{WCS}_{n, 1}$. We have the following two cases to be considered:

Subcase (i): if $W_{m} \nsubseteq\left\{k_{1}, k_{2}, k_{3}, \ldots, k_{n}\right\}$, then there must exist a vertex $l_{j}$ such that $l_{j} \in W_{m}$. Then, there exists at least one vertex $l_{i} \in W_{m}$ such that $k_{i-1}, k_{i+1} \notin W_{m}$. Then, for the corresponding edges $v k_{i-1}$ and $v k_{i+1}$, we have $r_{m}\left(v k_{i+1} \mid W_{m}\right)=r_{m}\left(v k_{i-1} \mid W_{m}\right)$, a contradiction. Therefore, $W_{m}$ is not a mixed resolving set for $\mathrm{WCS}_{n, 1}$ in this case.

Subcase (ii): if $W_{m} \subset\left\{k_{1}, k_{2}, k_{3}, \ldots, k_{n}\right\}$, then there exist at least two vertices $k_{i}$ and $k_{j}$ such that $k_{i}, k_{j} \notin W_{m}$. Then, for the edges $v k_{i}$ and $v k_{j}$, we have $r_{m}\left(v k_{i} \mid W_{m}\right)=r_{m}\left(v k_{j} \mid W_{m}\right)$, a contradiction. Therefore, $W_{m}$ is not a mixed resolving set for $\mathrm{WCS}_{n, 1}$ in this case as well. Thus, $\left|W_{m}\right| \geq 4 h+1$. This completes the proof for the second case.

For rest of the cases, the pattern is the same as that in Case (II).

\section{Mixed Metric Dimension of the Barycentric Subdivision of $W_{n, 1}$}

In this section, we determine the mixed metric dimension of the barycentric subdivision of a wheel graph.
5.1. Barycentric Subdivision of $W_{n, 1}$. Suppose $W_{n, 1}$ is a wheel graph with the vertex set $V\left(W_{n, 1}\right)=\left\{k_{1}, k_{2}, k_{3}, \ldots, k_{n}, v\right\}$ having a single universal vertex $v$. Now, each of the edges $k_{j} k_{j+1}$ and $v k_{j}(1 \leq j \leq n)$ of $W_{n, 1}$ is subdivided with a new vertex. The resulting graph so obtained is known as the barycentric subdivision wheel graph (BSWG) and is denoted by $\mathrm{WBS}_{n, 1}$. BSWG has $4 n$ edges, $E\left(\mathrm{WBS}_{n, 1}\right)=\left\{v l_{j}, l_{j} k_{j}\right.$, $\left.k_{j} m_{j}, m_{j} k_{j+1} \mid 1 \leq j \leq n\right\}$, and $3 n+1$ vertices, $V\left(\mathrm{WBS}_{n, 1}\right)=$ $\left\{v, l_{j}, k_{j}, m_{j} \mid 1 \leq j \leq n\right\}$, where all indices are taken to be modulo $n$ (see Figure 4 ). In this section, we obtain the mixed metric dimension of BSWG $\mathrm{WBS}_{n, 1}$.

Theorem 3. For $n \geq 6$, we have

$$
m \operatorname{dim}\left(\mathrm{WBS}_{n, 1}\right)= \begin{cases}4 h & \text { if } n=6 h, \\ 4 h+1 & \text { if } n=6 h+1 \\ 4 h+2 & \text { if } n=6 h+2 \\ 4 h+2 & \text { if } n=6 h+3 \\ 4 h+3 & \text { if } n=6 h+4 \\ 4 h+4 & \text { if } n=6 h+5\end{cases}
$$

Proof. To prove this, we first generate the mixed resolving sets for all the cases, obtaining the upper bounds depending on the positive integer $n$. Then, in the end, we show that the lower bound (or reverse inequality) is the same as the upper bound to conclude the theorem.

Case $(\mathrm{I}): n \equiv 0(\bmod 6)$. In this case, we have $n=6 h$, where $h \geq 2$ and $h \in \mathbb{N}$. Suppose an ordered subset $W_{m}=\left\{m_{1}, m_{2}, m_{4}, m_{5}, \ldots, m_{n-2}, m_{n-1}\right\}=\left\{m_{3 i+1}, m_{3 i+2}\right.$ $\mid 0 \leq i \leq 2 h-1\}$ of vertices in $\mathrm{WBS}_{n, 1}$ with $\left|W_{m}\right|=4 h$. Next, we claim that $W_{m}$ is the mixed resolving set for $\mathrm{WBS}_{n, 1}$. Now, we can give mixed codes to every vertex 


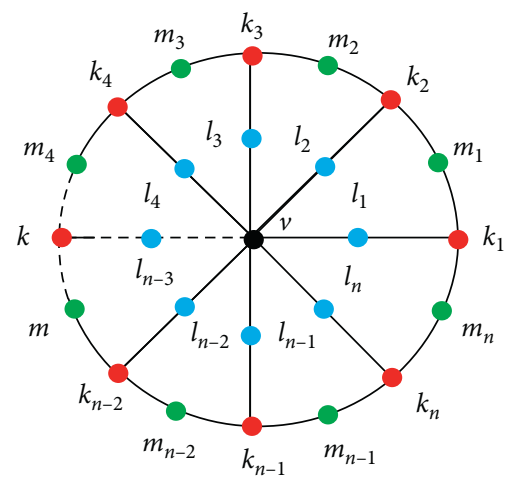

FIgURE 4: $\mathrm{WBS}_{n, 1}$.

and edge of $\mathrm{WBS}_{n, 1}$ with respect to $W_{m}$. The sets of mixed metric codes for the vertices $\left\{u=v, k_{j}, l_{j}\right.$, $\left.m_{j} \mid 1 \leq j \leq n\right\}$ of $\mathrm{WBS}_{n, 1}$ are as follows:

$$
\begin{aligned}
& A=\{r_{m}\left(v \mid W_{m}\right)=\underbrace{(3,3,3, \ldots, 3)}_{4 h \text {-times }}\}, \\
& B=\left\{\begin{array}{c}
r_{m}\left(k_{j} \mid W_{m}\right)=\left(\begin{array}{c}
5,5,5, \ldots, 5, d\left(m_{3 i+1}, k_{3 i+3}\right)=3, d\left(m_{3 i+2}, k_{3 i+3}\right)=1, \\
d\left(m_{3 i+4}, k_{3 i+3}\right)=5,5, \ldots, 5
\end{array}\right) \mid \\
j \equiv 0(\bmod 3) 0 \leq i \leq 2 h-1
\end{array}\right\} \cup \\
& \left\{r_{m}\left(k_{1} \mid W_{m}\right)=(1,3, \underbrace{5, \ldots, 5}_{(4 h-3) \text {-times }}, 3)\right\} \cup
\end{aligned}
$$

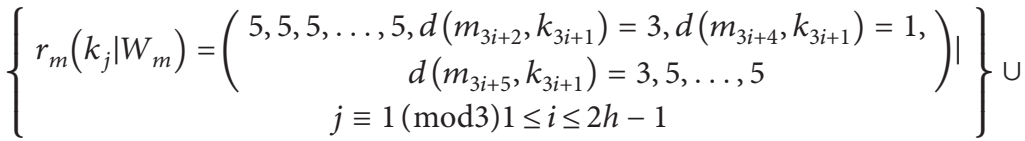

$$
\begin{aligned}
& \left\{\begin{array}{c}
r_{m}\left(k_{j} \mid W_{m}\right)=\left(5,5,5, \ldots, 5, d\left(m_{3 i+1}, k_{3 i+2}\right)=1, d\left(m_{3 i+2}, k_{3 i+2}\right)=1,5, \ldots, 5\right) \mid \\
j \equiv 2(\bmod 3) 0 \leq i \leq 2 h-1
\end{array}\right\}, \\
& C=\left\{\begin{array}{c}
r_{m}\left(l_{j} \mid W_{m}\right)=\left(4,, 4, \ldots, 4, d\left(m_{3 i+2}, l_{3 i+3}\right)=2,4, \ldots, 4\right) \mid \\
j \equiv 0(\bmod 3) 0 \leq i \leq 2 h-1
\end{array}\right\} \cup \\
& \left\{\begin{array}{c}
r_{m}\left(l_{j} \mid W_{m}\right)=\left(4,4,4, \ldots, 4, d\left(m_{3 i+1}, l_{3 i+1}\right)=2,4, \ldots, 4\right) \mid \\
j \equiv 1(\bmod 3) 0 \leq i \leq 2 h-1
\end{array}\right\} \cup \\
& \left\{\begin{array}{c}
r_{m}\left(l_{j} \mid W_{m}\right)=\left(4,4,4, \ldots, 4, d\left(m_{3 i+1}, l_{3 i+2}\right)=2, d\left(m_{3 i+2}, l_{3 i+2}\right)=2,4, \ldots, 4\right) \mid \\
j \equiv 2(\bmod 3) 0 \leq i \leq 2 h-1
\end{array}\right\},
\end{aligned}
$$

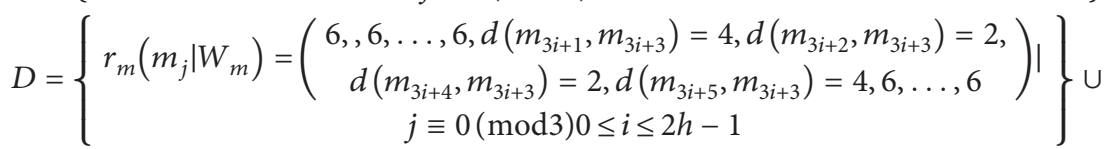

$$
\begin{aligned}
& \left\{r_{m}\left(m_{1} \mid W_{m}\right)=(0,2, \underbrace{6,6, \ldots, 6}_{(4 h-3) \text {-times }}, 4)\right\} U
\end{aligned}
$$

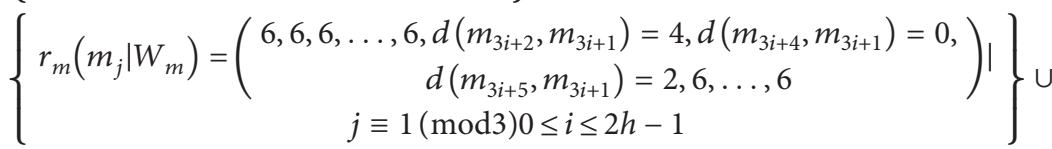

$$
\begin{aligned}
& \left\{r_{m}\left(l_{j} \mid W_{m}\right)=\left(\begin{array}{c}
6,6,6, \ldots, 6, d\left(m_{3 i+1}, m_{3 i+2}\right)=2, d\left(m_{3 i+2}, m_{3 i+2}\right)=0, \\
d\left(m_{3 i+4}, m_{3 i+2}\right)=4,6, \ldots, 6
\end{array}\right) \mid\right\} .
\end{aligned}
$$


Next, the sets of mixed metric codes for the edges $\left\{v l_{j}, l_{j} k_{j}, k_{j} m_{j}, m_{j} k_{j+1} \mid 1 \leq j \leq n\right\}$ of $\mathrm{WBS}_{n, 1}$ are as follows:

$$
\begin{aligned}
& E=\left\{\begin{array}{c}
r_{m}\left(v l_{j} \mid W_{m}\right)=\left(3,3,3, \ldots, 3, d\left(m_{3 i+2}, v l_{3 i+3}\right)=2,3, \ldots, 3\right) \mid \\
j \equiv 0(\bmod 3) 0 \leq i \leq 2 h-1
\end{array}\right\} \cup \\
& \left\{\begin{array}{c}
r_{m}\left(v l_{j} \mid W_{m}\right)=\left(3,3,3, \ldots, 3, d\left(m_{3 i+1}, v l_{3 i+1}\right)=2,3, \ldots, 3\right) \mid \\
j \equiv 1(\bmod 3) 0 \leq i \leq 2 h-1
\end{array}\right\} \cup \\
& \left\{\begin{array}{c}
r_{m}\left(v l_{j} \mid W_{m}\right)=\left(3,3,3, \ldots, 3, d\left(m_{3 i+1}, v l_{3 i+2}\right)=2, d\left(m_{3 i+2}, v l_{3 i+2}\right)=2,3, \ldots, 3\right) \mid \\
j \equiv 2(\bmod 3) 0 \leq i \leq 2 h-1
\end{array}\right\}, \\
& F=\left\{\begin{array}{c}
r_{m}\left(l_{j} k_{j} \mid W_{m}\right)=\left(\begin{array}{c}
4,4,4, \ldots, 4, d\left(m_{3 i+1}, l_{3 i+3} k_{3 i+3}\right)=3, d\left(m_{3 i+2}, l_{3 i+3} k_{3 i+3}\right)=1, \\
d\left(m_{3 i+4}, l_{3 i+3} k_{3 i+3}\right)=3,4, \ldots, 4 \\
j \equiv 0(\bmod 3) 0 \leq i \leq 2 h-1
\end{array}\right) \mid
\end{array}\right\} U \\
& \left\{r_{m}\left(l_{1} k_{1} \mid W_{m}\right)=(1,3, \underbrace{4,4, \ldots, 4}_{(4 h-3) \text {-times }}, 3)\right\} \cup \\
& \left\{r_{m}\left(l_{j} k_{j} \mid W_{m}\right)=\left(\begin{array}{c}
4,4,4, \ldots, 4, d\left(m_{3 i+2}, l_{3 i+1} k_{3 i+1}\right)=3, d\left(m_{3 i+4}, l_{3 i+1} k_{3 i+1}\right)=1, \\
d\left(m_{3 i+5}, l_{3 i+1} k_{3 i+1}\right)=3,4, \ldots, 4
\end{array}\right)|| U\right. \\
& \left\{\begin{array}{c}
r_{m}\left(l_{j} k_{j} \mid W_{m}\right)=\left(4,4,4, \ldots, 4, d\left(m_{3 i+1}, l_{3 i+2} k_{3 i+2}\right)=1, d\left(m_{3 i+2}, l_{3 i+2} l_{3 i+2}\right)=1,4, \ldots, 4\right) \mid \\
j \equiv 2(\bmod 3) 0 \leq i \leq 2 h-1
\end{array}\right\}, \\
& G=\left\{r_{m}\left(k_{j} m_{j} \mid W_{m}\right)=\left(\begin{array}{c}
5,5,5, \ldots, 5, d\left(m_{3 i+1}, k_{3 i+3} m_{3 i+3}\right)=3, d\left(m_{3 i+2}, k_{3 i+3} m_{3 i+3}\right)=1, \\
d\left(m_{3 i+4}, k_{3 i+3} m_{3 i+3}\right)=2, d\left(m_{3 i+5}, k_{3 i+3} m_{3 i+3}\right)=4,5, \ldots, 5 \\
j \equiv 0(\bmod 3) 0 \leq i \leq 2 h-1
\end{array}\right\} \cup\right. \\
& \left\{r_{m}\left(k_{1} m_{1} \mid W_{m}\right)=(0,2, \underbrace{5,5, \ldots, 5}_{(4 h-3)-\text { times }}, 3)\right\} \cup \\
& \left\{\begin{array}{c}
r_{m}\left(k_{j} m_{j} \mid W_{m}\right)=\left(\begin{array}{c}
5,5,5, \ldots, 5, d\left(m_{3 i+2}, k_{3 i+1} m_{3 i+1}\right)=3, d\left(m_{3 i+4}, k_{3 i+1} m_{3 i+1}\right)=0, \\
d\left(m_{3 i+5}, k_{3 i+1} m_{3 i+1}\right)=2,5, \ldots, 5
\end{array}\right) \mid \\
j \equiv 1(\bmod 3) 1 \leq i \leq 2 h-1
\end{array}\right\} \cup \\
& \left\{\begin{array}{c}
r_{m}\left(k_{j} m_{j} \mid W_{m}\right)=\left(\begin{array}{c}
5,5,5, \ldots, 5, d\left(m_{3 i+1}, k_{3 i+2} m_{3 i+2}\right)=1, d\left(m_{3 i+2}, k_{3 i+2} m_{3 i+2}\right)=0, \\
d\left(m_{3 i+4}, k_{3 i+2} m_{3 i+2}\right)=4,5, \ldots, 5
\end{array}\right) \mid \\
j \equiv 2(\bmod 3) 0 \leq i \leq 2 h-1
\end{array}\right\}, \\
& H=\left\{r_{m}\left(m_{j} k_{j+1} \mid W_{m}\right)=\left(\begin{array}{c}
5,5,5, \ldots, 5, d\left(m_{3 i+1}, m_{3 i+3} k_{3 i+4}\right)=4, d\left(m_{3 i+2}, m_{3 i+3} k_{3 i+4}\right)=2, \\
d\left(m_{3 i+4}, m_{3 i+3} k_{3 i+4}\right)=1, d\left(m_{3 i+5}, m_{3 i+3} k_{3 i+4}\right)=3,5, \ldots, 5 \\
j \equiv 0(\bmod 3) 0 \leq i \leq 2 h-1
\end{array}\right\} \mid\right. \\
& \left\{r_{m}\left(m_{1} k_{2} \mid W_{m}\right)=(0,1, \underbrace{5,5, \ldots, 5}_{(4 h-3)-\text { times }}, 4)\right\} U \\
& \left\{\begin{array}{c}
r_{m}\left(m_{j} k_{j+1} \mid W_{m}\right)=\left(\begin{array}{c}
5,5,5, \ldots, 5, d\left(m_{3 i+2}, m_{3 i+1} k_{3 i+2}\right)=4, d\left(m_{3 i+4}, m_{3 i+1} k_{3 i+2}\right)=0, \\
d\left(m_{3 i+5}, m_{3 i+1} k_{3 i+2}\right)=1,5, \ldots, 5
\end{array}\right) \mid \\
j \equiv 1(\bmod 3) 1 \leq i \leq 2 h-1
\end{array}\right\} \cup \\
& \left\{\begin{array}{c}
r_{m}\left(m_{j} k_{j+1} \mid W_{m}\right)=\left(\begin{array}{c}
5,5,5, \ldots, 5, d\left(m_{3 i+1}, m_{3 i+2} k_{3 i+3}\right)=2, d\left(m_{3 i+2}, m_{3 i+2} k_{3 i+3}\right)=0, \\
d\left(m_{3 i+4}, m_{3 i+2} k_{3 i+3}\right)=3,5, \ldots, 5
\end{array}\right) \mid \\
j \equiv 2(\bmod 3) 0 \leq i \leq 2 h-1
\end{array}\right\} .
\end{aligned}
$$


From these sets of mixed codes for $\mathrm{WBS}_{n, 1}$, we obtain that $|A|=1,|B|=|C|=|D|=|E|=|F|=|G|=|H|=n$, and $A \cap B \cap C \cap D \cap E \cap F \cap G \cap H=\varnothing$, implying $W_{m}$ to be a mixed resolving set for $\mathrm{WBS}_{n, 1}$, i.e., $m \mathrm{dim}$ $\left(\mathrm{WBS}_{n, 1}\right) \leq 4 h$. Next, using equation (1) and Proposition 2 , we find that $m \operatorname{dim}\left(\mathrm{WBS}_{n, 1}\right)=4 h$, in this case.

Like the first case, the rest of the proof is similar to that of Theorem 2.

Remark 2. For the cycle and barycentric subdivision wheel graph, i.e., $H=\mathrm{WCS}_{n, 1}$ and $H=\mathrm{WBS}_{n, 1}$, we find that $\operatorname{dim}(H)=e \operatorname{dim}(H)=m \operatorname{dim}(H) \quad$ when $n=6 h \quad$ and $n=6 h+3$. For the rest of the values of the positive integer $n$, we have $\operatorname{dim}(H)=e \operatorname{dim}(H)<m \operatorname{dim}(H)$ (using Propositions 2 and 4 and Theorems 2 and 3 ).

\section{Conclusion}

In this article, we have computed the mixed metric dimension for three families of graphs, namely, $\mathrm{WBS}_{n, 1}$, $\mathrm{WCS}_{n, 1}$, and $\mathrm{WSS}_{n, 1}$, obtained after the barycentric, cycle, and spoke subdivisions of the wheel graph $W_{n, 1}$, respectively. We also observed that the mixed resolving sets for $\mathrm{WBS}_{n, 1}$ and $\mathrm{WCS}_{n, 1}$ are independent. For $\mathrm{WSS}_{n, 1}$, we found that $\operatorname{dim}\left(\mathrm{WSS}_{n, 1}\right)<e \operatorname{dim}\left(\mathrm{WSS}_{n, 1}\right)<m \operatorname{dim}\left(\mathrm{WSS}_{n, 1}\right)$, and for $H=$ $\mathrm{WBS}_{n, 1}$ and $H=\mathrm{WCS}_{n, 1}$, we obtained the following relation: $\operatorname{dim}(H)=e \operatorname{dim}(H) \leq m \operatorname{dim}(H) \quad$ (partial answers to the questions raised in $[1,18])$.

\section{Data Availability}

Data sharing is not applicable to this article as no datasets were generated or analyzed during the current study.

\section{Conflicts of Interest}

The authors declare no conflicts of interest.

\section{Authors' Contributions}

All the authors contributed equally to the final manuscript.

\section{Acknowledgments}

This research was supported by the Natural Science Foundation of China (11871077) and the NSF of Anhui Province (1808085MA04).

\section{References}

[1] A. Kelenc, D. Kuziak, A. Taranenko, and I. G. Yero, "Mixed metric dimension of graphs," Applied Mathematics and Computation, vol. 314, pp. 429-438, 2017.

[2] P. J. Slater, “Abstracts,” Stroke, vol. 6, no. 5, pp. 549-559, 1975.

[3] F. Harary and R. A. Melter, "On the metric dimension of a graph,” ARS Combinatoria, vol. 2, pp. 191-195, 1976.

[4] M. Azeem and M. F. Nadeem, "Metric-based resolvability of polycyclic aromatic hydrocarbons," The European Physical Journal Plus, vol. 136, no. 4, pp. 1-14, 2021.
[5] G. Chartrand, L. Eroh, M. A. Johnson, and O. R. Oellermann, "Resolvability in graphs and the metric dimension of a graph," Discrete Applied Mathematics, vol. 105, no. 1-3, pp. 99-113, 2000.

[6] Z. Hussain, M. Munir, A. Ahmad, M. Chaudhary, J. A. Khan, and I. Ahmed, "Metric basis and metric dimension of 1pentagonal carbon nanocone networks," Scintific Reports, vol. 10, no. 1, pp. 1-7, 2020.

[7] B. Deng, M. F. Nadeem, and M. Azeem, "On the edge metric dimension of different families of möbius networks," Mathematical Problems in Engineering, vol. 2021, Article ID 6623208, 9 pages, 2021.

[8] S. Khuller, B. Raghavachari, and A. Rosenfeld, "Landmarks in graphs," Discrete Applied Mathematics, vol. 70, no. 3, pp. 217-229, 1996.

[9] P. Singh, S. Sharma, S. K. Sharma, and V. K. Bhat, "Metric dimension and edge metric dimension of windmill graphs," AIMS Mathematics, vol. 6, no. 9, pp. 9138-9153, 2021.

[10] H. Raza and Y. Ji, "Computing the mixed metric dimension of a generalized petersen graph $P(n, 2)$," Frontiers of Physics, vol. 8211 pages, 2020.

[11] A. Sebo and E. Tannier, "On metric generators of graphs," Mathematics of Operations Research, vol. 29, no. 2, pp. 383393, 2004.

[12] S. K. Sharma and V. K. Bhat, "Metric Dimension of heptagonal circular ladder," Discrete Mathematics, Algorithms and Applications, vol. 13, no. 1, Article ID 2050095, 2021.

[13] S. K. Sharma and V. K. Bhat, "Fault-tolerant metric dimension of two-fold heptagonal-nonagonal circular ladder," Discrete Mathematics, Algorithms and Applications, Article ID 2150132, 2021.

[14] M. F. Nadeem, M. Azeem, and A. Khalil, "The locating number of hexagonal Möbius ladder network," Journal of Applied Mathematics and Computing, vol. 66, no. 1, pp. 149-165, 2021.

[15] G. Chartrand, V. Saenpholphat, and P. Zhang, "The independent resolving number of a graph," Mathematica Bohemica, vol. 128, no. 4, pp. 379-393, 2003.

[16] F. Okamoto, B. Phinezy, and P. Zhang, "The local metric dimension of a graph," Mathematica Bohemica, vol. 135, no. 3, pp. 239-255, 2010.

[17] C. Hernando, M. Mora, P. J. Slater, and D. R. Wood, "Faulttolerant metric dimension of graphs," Convexity in Discrete Structures, vol. 5, pp. 81-85, 2008.

[18] A. Kelenc, N. Tratnik, and I. G. Yero, "Uniquely identifying the edges of a graph: the edge metric dimension," Discrete Applied Mathematics, vol. 251, pp. 204-220, 2018.

[19] H. Raza, J. B. Liu, and S. Qu, "On mixed metric dimension of rotationally symmetric graphs," IEEE Access, vol. 8, pp. 11560-11569, 2019.

[20] H. Raza, Y. Ji, and S. Qu, "On mixed metric dimension of some path related graphs," IEEE Access, vol. 8, pp. 188146188153, 2020.

[21] Z. Raza and M. S. Bataineh, "The comparative analysis of metric and edge metric dimension of some subdivisions of the wheel graph," Asian-European Journal of Mathematics, vol. 14, no. 98, Article ID 2150062, 2020.

[22] I. Tomescu and A. Riasat, "On metric dimension of uniform subdivisions of the wheel," Utilitas Mathematica, vol. 96, pp. 233-242, 2015.

[23] I. Tomescu and I. Javaid, "On the metric dimension of the Jahangir graph," Bulletin Mathématique de la Société des Sciences Mathématiques de Roumanie, vol. 50, no. 98, pp. 371-376, 2007. 